\title{
Thermodynamically Improbable Phase Diagrams
}

\author{
Hiroaki Okamoto \\ ASM International \\ Materials Park, OH 44073 \\ and \\ T.B. Massalski \\ Carnegie Mellon University \\ Pittsburgh, PA 15213-3890
}

\begin{abstract}
Phase diagrams showing very unlikely boundaries, while not explicitly violating thermodynamic principles or phase rules, are discussed. Phase rule violations in proposed phase diagrams often become apparent when phase boundaries are extrapolated into metastable regions. In addition to phase rule violations, this article considers difficulties regarding an abrupt change of slope of a phase boundary, asymmetric or unusually pointed liquidus boundaries, location of miscibility gaps, and gas/liquid equilibria. Another frequent source of phase diagram errors concerns the initial slopes of liquidus and solidus boundaries in the very dilute regions near the pure elements. Useful and consistent prediction can be made from the application of the van't Hoff equation for the dilute regions.
\end{abstract}

\section{Introduction}

In the course of editing phase diagrams for the Second Edition of Binary Alloy Phase Diagrams, we discovered numerous phase diagrams that showed very unlikely phase boundaries in various respects although they did not explicitly violate phase rules. This article discusses several of the unlikely phase diagram features encountered. Explicit violations of phase rules are briefly reviewed first, followed by implicit cases of possible phase rule violations and some more subtle phase boundary features that may come under question when constructing phase diagrams is discussed in detail.

\subsection{Typical Phase Rule Violations}

When a suggested phase diagram is examined, obvious violations of phase rules and other thermodynamic principles are usually checked first to confirm that the proposed phase diagram representations are generally valid. A hypothetical phase diagram (Fig. 1) illustrates such typical violations at points $A$ to $T$. The potential problems encountered at each point are superficially described below. Thermodynamically rigorous explanation of these problems are contained in standard textbooks and articles (e.g., [56Rhi], [66Pri], [68Gor], and [81Goo]). Most of these problems can also be demonstrated graphically with the use of appropriate free energy curves.

A: A two-phase field cannot be extended to become part of a pure element side of a phase diagram at zero solute. In example $A$, the liquidus and the solidus must meet at the melting point of the pure element.

$B$ : Two liquidus curves must meet at one composition at a eutectic temperature.

$C:$ A tie line must terminate at a phase boundary.

$D$ : Two solvus boundaries (or two liquidus, or two solidus, or a solidus and a solvus) of the same phase must meet (i.e., intersect) at one composition at an invariant temperature. (There should not be two solubility values for a phase boundary at one temperature.)

$E$ : A phase boundary must extrapolate into a two-phase field after crossing an invariant point. The validity of this feature, and similar features related to invariant temperatures, is easily demonstrated by constructing hypothetical free energy diagrams slightly below and slightly above the invariant temperature and by observing the relative positions of the relevant tangent points to the free energy curves. After intersection, such boundaries can also be extrapolated into metastable regions of the phase diagram. Such extrapolations are sometimes indicated by dashed or dotted lines.

$F$ : Two single-phase fields ( $\alpha$ and $\beta$ ) should not be in contact along a horizontal line. (An invariant temperature line separates two-phase fields in contact.)

$G$ : Asingle-phase field ( $\alpha$ in this case) should not be apportioned into subdivisions by a single line. Having created a horizontal (invariant) line at $F$ (which is an error), there may be a temptation to extend this line into a single-phase field, $\alpha$, creating an additional error.

$H$ : In a binary system, an invariant temperature line should involve equilibrium among three phases.

I: There should be a two-phase field between two single-phase fields. (Two single phases cannot touch except at a point. However, second-order and higher-order transformations may be exceptions to this rule.)

$J$ : When two phase boundaries touch at a point, they should touch at an extremity of temperature.

$K$ : A touching liquidus and solidus (or any two touching boundaries) must have a horizontal common tangent at the congruent point. In this case, the solidus at the melting point is too "sharp" and appears to be discontinuous. 
L: Alocal minimum point in the lower part of a single-phase field (in this case the liquid) cannot be drawn without an additional boundary in contact with it. (In this case, a horizontal monotectic line is most likely missing.)

$M:$ A local maximum point in the lower part of a single-phase field cannot be drawn without a monotectic, monotectoid, syntectic, and sintectoid reaction occurring below it at a lower temperature. Alternatively, a solidus curve must be drawn to touch the liquidus at point $M$.

$N$ : Alocal maximum point in the upper part of a single-phase field cannot be drawn without the phase boundary touching a reversed monotectic, or a monotectoid, horizontal reaction line coinciding with the temperature of the maximum. When an $N$-type error is introduced, a minimum may be created on either side (or on one side) of $N$. This introduces an additional error, which is the opposite of $M$, but equivalent to $M$ in kind.

$O$ : A phase boundary cannot terminate within a phase field. (Termination due to lack of data is, of course, often shown in phase diagrams, but this is recognized to be artificial.)

$P$ : The temperature of an invariant reaction in a binary system must be constant. (The reaction line must be horizontal.)

$Q:$ The liquidus should not have a discontinuous sharp peak at the melting point of a compound. (This rule is not applicable if the liquid retains the molecular state of the compound, i.e., in case of an ideal association.)

$R$ : The compositions of all three phases at an invariant reaction must be different.

$S:$ A four-phase equilibrium is not allowed in a binary system.
T: Two separate phase boundaries that create a two-phase field between two phases in equilibrium should not cross one another.

\subsection{Problems Connected with Phase Boundary Curvatures}

Although phase rules are not violated, three additional unusual situations $(X, Y$, and $Z)$ have also been included in Fig. 1. In each case, a more subtle thermodynamic problem may exist related to these situations. Examples will be discussed later when several thermodynamically unlikely diagrams are considered below. The problems with each of these situations involve an indicated rapid change of slope of a phase boundary. If $X$-, $Y$, and $Z$-type situations are to be associated with realistic thermodynamics, the temperature (or the composition) dependence of the thermodynamic functions of the phase (or phases) involved would be expected to show corresponding abrupt and unrealistic variations in the phase diagram regions where such abrupt phase boundary changes are proposed, without any clear reason for them. Even the onset of ferromagnetism in a phase does not normally cause an abrupt change of slope of the related phase boundaries. The unusual changes of slope considered here are $X, Y$, and $Z$.

$X$ : Two inflection points are located too closely to one another.

Y: An abrupt reversal of the boundary direction (more abrupt than a typical smooth "retrograde"). This particular change can occur only if there is an accompanying abrupt change in the temperature dependence of the thermodynamic properties of either of the two phases involved (in this case $\delta$ or $\lambda$ in relation to the boundary). The boundary turn at $Y$ is very unlikely to be explained by any realistic change in the composition dependence of the Gibbs energy functions.

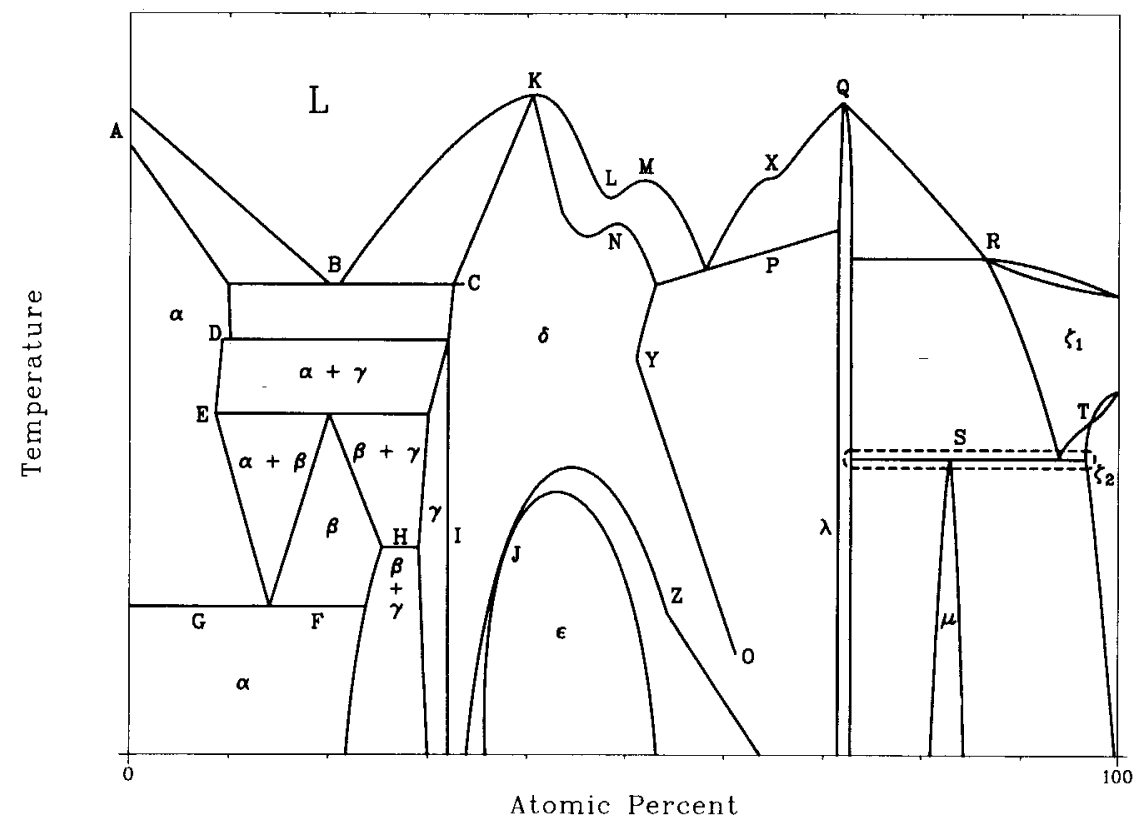

Fig. 1 Hypothetical phase diagram showing violations of phase rules and thermodynamically impossible situations (points $A-T$ ). Very unlikely changes of slopes are also added (points $X-Z$ ). 
$Z$ : An abrupt change in the slope of a single phase boundary. This particular change can occur only by an abrupt change in the composition dependence of the thermodynamic properties of the single phase involved (in this case the $\delta$ phase). It cannot be explained by any possible abrupt change in the temperature dependence of the Gibbs energy function of the phase. (If the temperature-dependence were involved, there would also be a change in the boundary of the $\varepsilon$ phase.)

In Fig. a1 of the Appendix, Fig. 1 is redrawn removing all the errors illustrated in that figure. We emphasize that other solutions can be proposed to remove some of the errors. Figure a1 merely serves as an example of an error-free diagram.

\section{Less Obvious Phase Rule Violations}

Errors $A$ to $T$ in Fig. 1 are often encountered in explicit forms in numerous published binary phase diagrams. Some errors, such as $E, J, K$, and $Q$, are quite common, and are sometimes introduced inadvertently in the drafting stage. Besides these explicit errors, many questionable phase diagram regions exist in proposed phase diagrams which, although they seemingly do not violate any of the above rules, are nevertheless very unlikely to be true phase representations. Some of these questionable situations become evident only when the respective phase boundaries are extrapolated to the metastable equilibrium region. In performing the extrapolation, it often becomes evident that only through a strange or an abrupt (and hardly justified) change of slope, leading to $X$-, $Y$-, and $Z$-type errors, could the proposed phase diagram be reconciled in the metastable regions without committing the $A$ to $T$ errors. We illustrate these difficulties with some specific situations described below. In each situation, there may be alternative interpretations (i.e, corrections) of the indicated error and how to remove it. However, only selected possibilities and remedies are considered in this article.

\subsection{Problems Related to the A-Type Error}

The proposed Ce-Pr phase diagram (Fig. 2) [82Gsc] involves an $A$-type problem. Assuming that the trend of the $[(\gamma \mathrm{Ce})+$ $(\beta \mathrm{Ce}, \alpha \mathrm{Pr})] /(\beta \mathrm{Ce}, \alpha \mathrm{Pr})$ solvus is correct, its extrapolation to the Pr-rich side appears to cross the 100 at. $\%$ Pr line at $\sim 800^{\circ} \mathrm{C}$. Be- cause no two-phase fields should be above this temperature at 100 at.\% $\mathrm{Pr}$, also in the metastable state, an extrapolation of the $(\gamma \mathrm{Ce}) /[(\gamma \mathrm{Ce})+(\beta \mathrm{Ce}, \alpha \mathrm{Pr})]$ solvus should cross the 100 at.\% $\mathrm{Pr}$ line at the same temperature. However, this requires a rather unlikely and abrupt change of slope in the latter solvus. Alternatively, if the latter solvus is correct, the former solvus must be modified so that it does not cross the 100 at.\% Pr line at all when extrapolated smoothly. At any rate, either one or the other of the solvus boundaries appears to need revision.

\subsection{Problems Related to the $\mathbf{Q}$-Type Error}

In the proposed $\mathrm{Cr}$-C phase diagram (Fig. 3) [58Han], $\mathrm{Cr}_{7} \mathrm{C}_{3}$ and $\mathrm{Cr}_{3} \mathrm{C}_{2}$ melt peritectically. When the liquidus of $\mathrm{Cr}_{7} \mathrm{C}_{3}$ is extrapolated smoothly toward the $\mathrm{Cr}_{7} \mathrm{C}_{3}$ compound, the projected metastable congruent melting maximum occurs at a composition somewhere between $\mathrm{Cr}_{7} \mathrm{C}_{3}$ and $\mathrm{Cr}_{3} \mathrm{C}_{2}$. Because the congruent melting point must occur at the $\mathrm{Cr} \gamma \mathrm{C}_{3}$ composition, the extrapolated liquidus must have an unexpectedly sharp drop past the needed maximum at $\mathrm{Cr}_{7} \mathrm{C}_{3}$, causing a possible $Q$-type problem (i.e., a discontinuity) in the metastable range. A similar situation also occurs for $\mathrm{Cr}_{3} \mathrm{C}_{2}$. Because the liquidus curve should have a horizontal and a continuous slope at the congruent melting temperature when extrapolated, the correct liquidi of $\mathrm{Cr}_{7} \mathrm{C}_{3}$ and $\mathrm{Cr}_{3} \mathrm{C}_{2}$ when projected in Fig. 3 should be nearly horizontal at the temperatures near the projected respective metastable melting points. In the $\mathrm{Cr}$-C system, this is also further complicated by the indicated positions of the two compounds, which are each very close to the intersection of the horizontal invariant temperature lines and the liquidus boundaries, as indicated in the diagram. The liquidus does indeed make a sharp turn in the metastable range on one side of a compound in the vicinity of a congruent melting point without introducing a $Q$-type violation. However, if a potential $Q$-type problem is thereby ignored, a $Y$-type problem may have to be considered instead. These relatively minor errors in the $\mathrm{Cr}-\mathrm{C}$ system are not repeated in the new diagram proposed by [90Ven2].

\subsection{Problems Related to the T-Type Error}

The $T$-type error is so obvious that it is virtually unseen in an explicit form in the majority of the published phase diagrams. How-

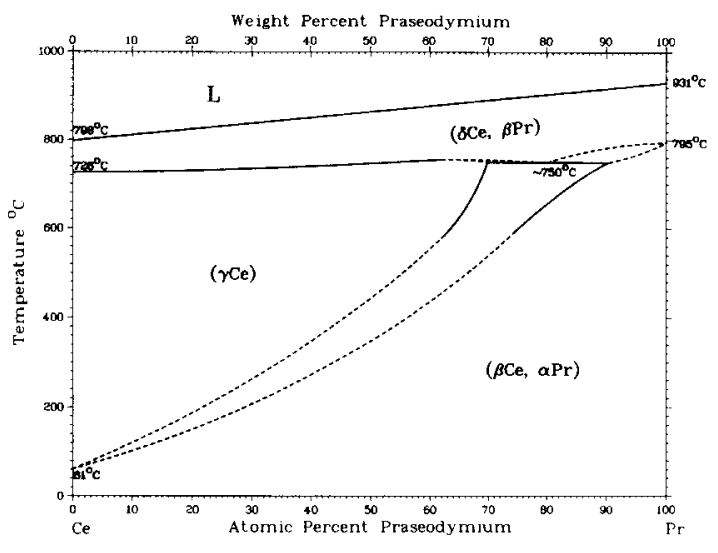

Fig. 2 Ce-Pr phase diagram. Redrawn from [82Gsc].

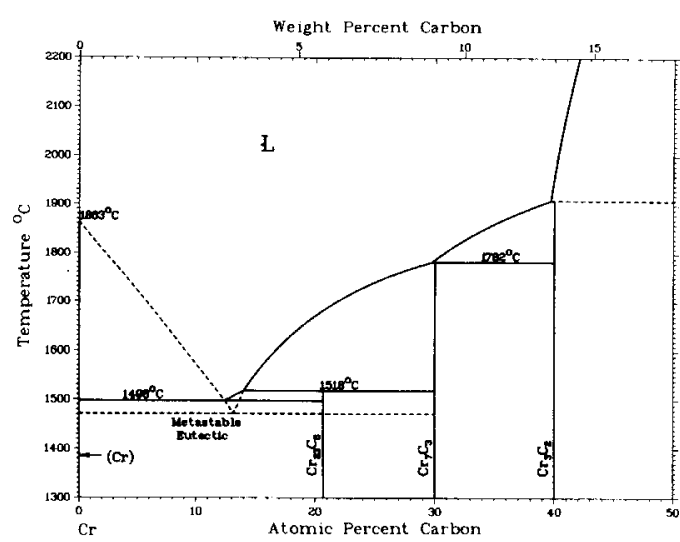

Fig. $3 \mathrm{Cr}$-C phase diagram. Redrawn from [58Han]. 


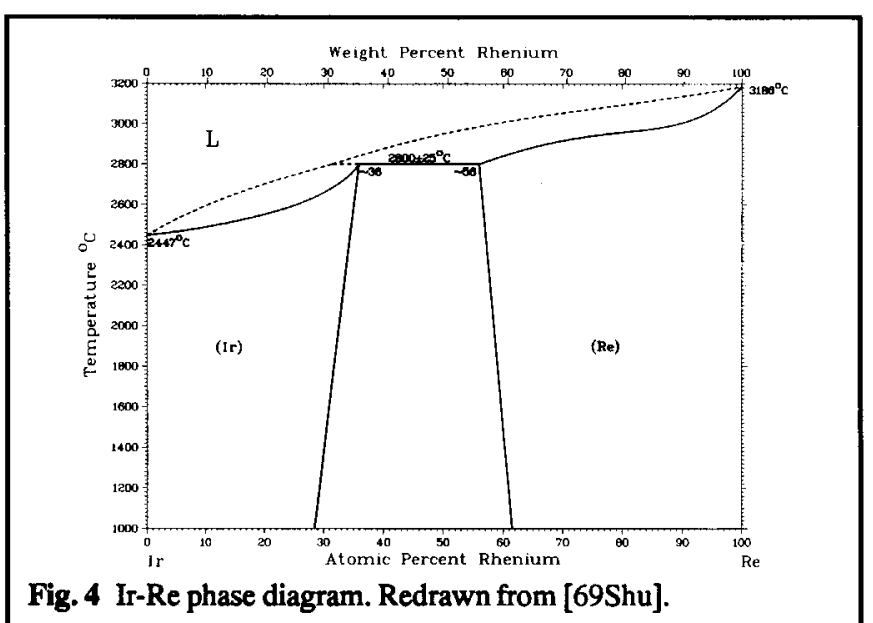

ever, many diagrams with two-phase boundaries show trends that would form a $T$-type error if extrapolated to the metastable range. For example, the upper and lower boundaries of the L+ (Ir) twophase field in the Ir-Re phase diagram (Fig. 4) [69Shu] would cross one another when extrapolated smoothly to the Re-rich side. If this were not to happen, an abrupt change of slope would be required in a narrow composition range (violation of the $X$ or $Z$ type). Hence, the proposed shape of the two-phase field in this system is probably inaccurate.

\subsection{Problems Related to the $Y$ - or Z-Type Errors}

An abrupt change of slope in a phase boundary, of the $Y$ - or $Z$-type (Fig. 1), that may actually be seen in a proposed phase diagram is usually caused by a drafting error. However, for such an abrupt change of slope to be real, the thermodynamic properties of the given phase would have to change quite abruptly at that particular temperature or composition. As shown below, an abrupt change of a thermodynamic property, without being accompanied by a first-order phase transition, is rather unlikely.

If a kink is observed on a liquidus in a temperature-composition phase diagram, as in Fig. 5 at point $p$, then an accompanying abrupt change must exist in either: (1) the temperature-dependence of the lattice stability parameter of the element $A$ (or compound) at temperature $a$, or (2) an abrupt change in the temperature-dependence of the Gibbs energy of mixing of the liquid phase $\Delta_{\operatorname{mix}} G(L)$, or (3) an abrupt change in its composition dependence. For situation (1), if the element $A$ undergoes a phase transformation at temperature $a, p$ could be an expected boundary change. However, the liquidus would then consist of two separate segments and a horizontal tie line ap would have to exist. A significant change in the lattice stability parameter of an element can be expected if there exists a Curie temperature transition (ideally, $\Delta C_{p}=\infty$ ). However, even then, the change of slope in the boundary of a phase in equilibrium with the ferromagnetic element (such as Fe), or a ferromagnetic compound in the phase diagram, often turns out to be gradual over the Curie temperature range (see e.g., [81Nis]). A similar situation also has been confirmed for the smooth phase boundary of the $\alpha$ phase in the $\mathrm{Cu}-\mathrm{Zn}$ system, in the region of the order-disorder transition occurring in the bordering $\beta$ phase (which is a higher-order transition) [57Bec].

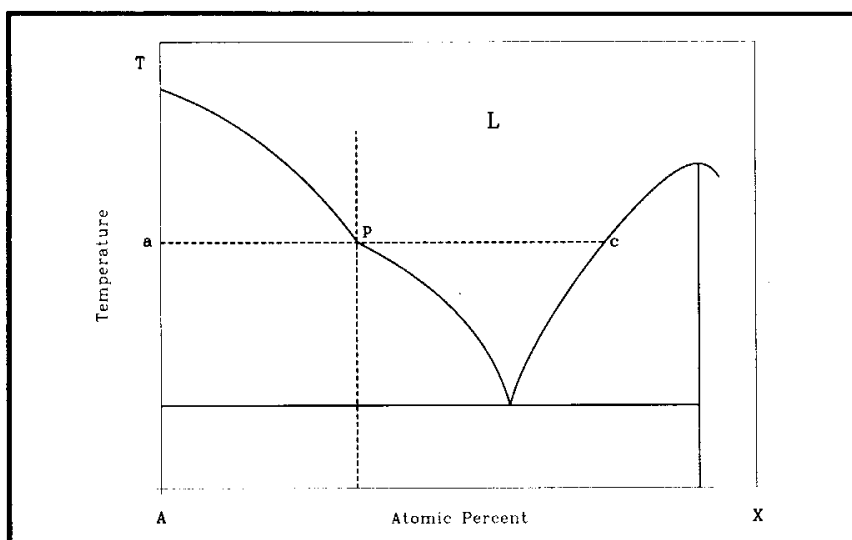

Fig. 5 Hypothetical phase diagram with an abrupt change of slopes in the $\mathrm{L} /(\mathrm{L}+A)$ liquidus at point $p$.

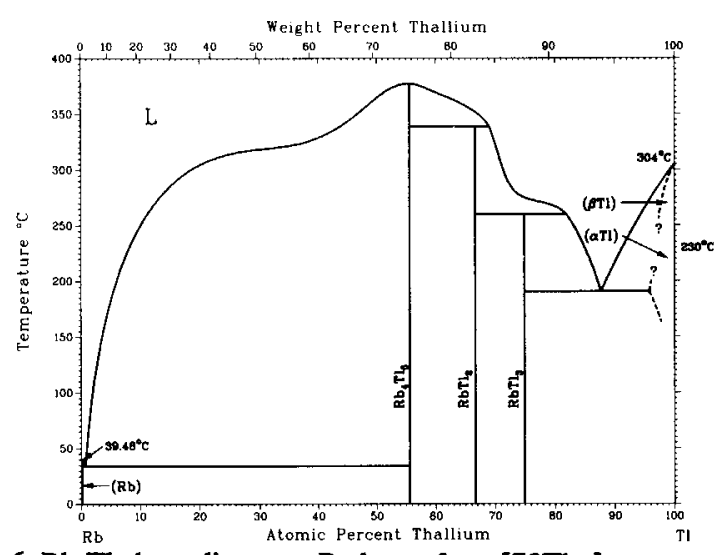

Fig. $6 \mathrm{Rb}-\mathrm{Tl}$ phase diagram. Redrawn from [70Thu].

If the kink at $p$ is due to an abrupt change in the temperaturedependence of $\Delta_{\mathrm{mix}} G(\mathrm{~L})$, there must be an accompanying boundary change at point $c$ (Fig. 5). In this hypothetical diagram, to obtain an abrupt change in the temperature-dependence of $\Delta_{\mathrm{mix}} G(\mathrm{~L})$, the specific heat $C_{p}$ of the liquid phase would have to change abruptly. However, the temperature-dependence of $C_{p}$ is almost always a gradual function within a given phase. Finally, if the kink at $p$ were due to an abrupt change in the composition-dependence of $\Delta_{\text {mix }} G(\mathrm{~L})$, the interaction energy between the two elements of the binary system in the region in question would have to be changing abruptly without an obvious reason.

Admittedly, the above discussion involves qualitative arguments, and there remains the important question of "how abrupt is abrupt?" when changes of slope, kinks, twists, and other features of phase boundaries are examined. Probably, the behavior of the second derivative (curvature) of the phase boundary trend is a good indicator of the abruptness. For example, an extrapolation of the liquidus of $\mathrm{RbTl}_{3}$ above its peritectic melting point in the binary Rb-T1 diagram (Fig. 6) [70Thu] requires a very abrupt change of curvature above the peritectic temperature. The liquidus temperature of $\mathrm{RbTl}_{3}$ must fall below that of $\mathrm{RbTl}_{2}$ above the peritectic temperature (to avoid the $E$-type error in Fig. 1), and the liquidus of $\mathrm{RbTl}_{3}$ must have a peak at the composition of $\mathrm{RbTl}_{3}$ when extrapolated. As a result, the needed curvature change be- 
comes very pronounced only near the peritectic temperature (Fig. 7). In the above example, if an ideal liquid is assumed, the required numerical free energy change for the Gibbs energy of $\mathrm{RbTl}_{3}$ (with respect to liquid $\mathrm{Rb}$ and liquid $\mathrm{Tl}$ ) is $-3660+2.063 T$ $\mathrm{J} / \mathrm{mol}$ above the peritectic temperature and $-1420-2.139 T \mathrm{~J} / \mathrm{mol}$
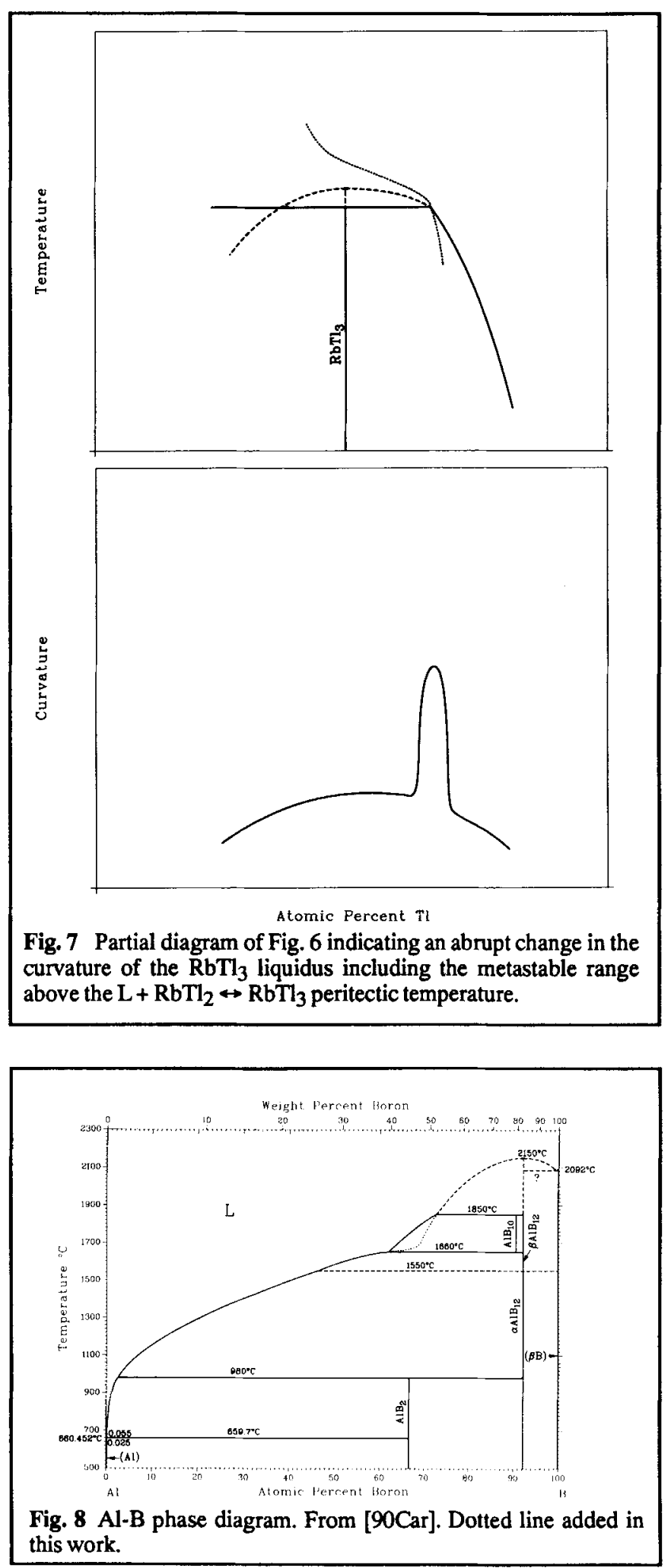

below the peritectic temperature. The change from one form of the Gibbs energy function to the other form in a narrow temperature range appears to be unjustified and unreasonable. Thus, in the $\mathrm{Rb}-\mathrm{Tl}$ case, the form of the $\mathrm{RbTl}_{2}$ liquidus is most likely in error.

In the Al-B phase diagram [90Car] (Fig. 8), a similar problem occurs in the metastable range. The liquidus of $\beta A \mathrm{BB}_{12}$ consists of two segments $\left(2150\right.$ to $1850^{\circ} \mathrm{C}$ and 1660 to $1550^{\circ} \mathrm{C}$ ) on the $\mathrm{Al}-$ rich side. These upper and lower segments must be continuous in the metastable range, because there is no reported phase transformation in $\mathrm{AlB}_{12}$ between 1660 and $1850^{\circ} \mathrm{C}$. However, a connection through extrapolation of the two liquidus segments (dotted line) causes a $Y$-type problem (requiring an abrupt change in the Gibbs energy of $A l B_{12}$ ). Since the liquidus of $A_{1 B} B_{10}$ and the lower branch of the $B \mathrm{AlB}_{12}$ liquidus appear to be well established (solid lines), the most likely error is in the upper branch of the $\beta_{A l B}$ liquidus. Unfortunately, any modification of this boundary does not improve the situation unless a slight upward modification of the lower liquidus is also added. The indicated projection of the upper liquidus towards the $\mathrm{B}$ side is also questionable here because of a possible $A$-type problem as discussed below.

The Al-Se phase diagram (Fig.9) [89How] also shows a situation that requires an abrupt change of slope to accommodate the proposed phase boundaries. If extrapolated smoothly to the $\mathrm{Al}$ side beyond the $\mathrm{L} \leftrightarrow(\mathrm{Al})+\mathrm{Al}_{2} \mathrm{Se}_{3}$ eutectic, the $\mathrm{L}\left(\mathrm{L}+\mathrm{Al}_{2} \mathrm{Se}_{3}\right)$ liquidus is projected to cross the 0 at.\% Se line. However, this liquidus (which is with respect to the $\mathrm{Al}_{2} \mathrm{Se}_{3}$ phase) must not be extrapolated to cross the 0 at.\% Se line. If this were assumed to be possible, the pure $A l$ side would include the $\mathrm{Al}+\mathrm{Al}_{2} \mathrm{Se}_{3}$ twophase field below the intersection temperature of the extrapolated liquidus (a clear $A$-type violation). The slope of the Gibbs free energy of mixing in the liquid phase must be $-\infty$ at 0 at.\% Se because of the $R T X \ln X$ term in the entropy of mixing. Consequently, the $\left.\mathrm{L} / \mathrm{L}+\mathrm{Al}_{2} \mathrm{Se}_{3}\right)$ liquidus (including the extrapolated part) can meet the 0 at.\% Se line only at $0 \mathrm{~K}$. It follows that the shape of the liquidus near the $\mathrm{Al}$ side must be modified to be nearly vertical already at the eutectic. A slightly changed position of the eutectic could probably also improve the situation, if it could be adjusted, from essentially 0 at.\% Se to a finite composition. The same problem occurs at the Se side of this phase diagram.

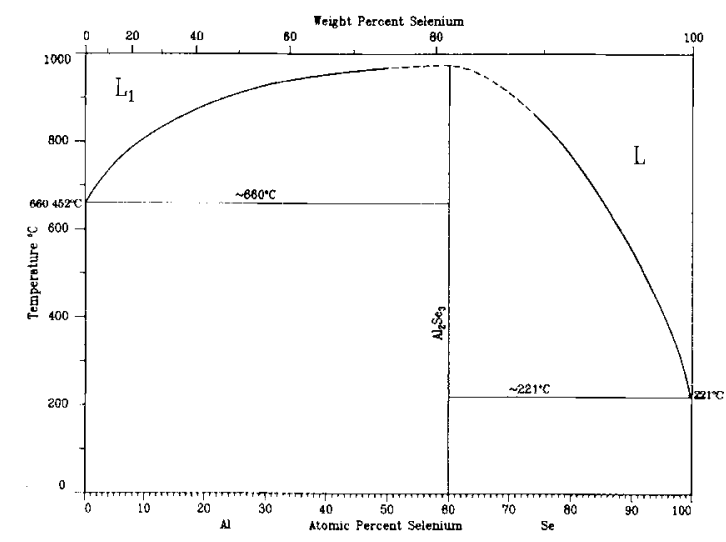

Fig. 9 Al-Se phase diagram. From [89How]. 


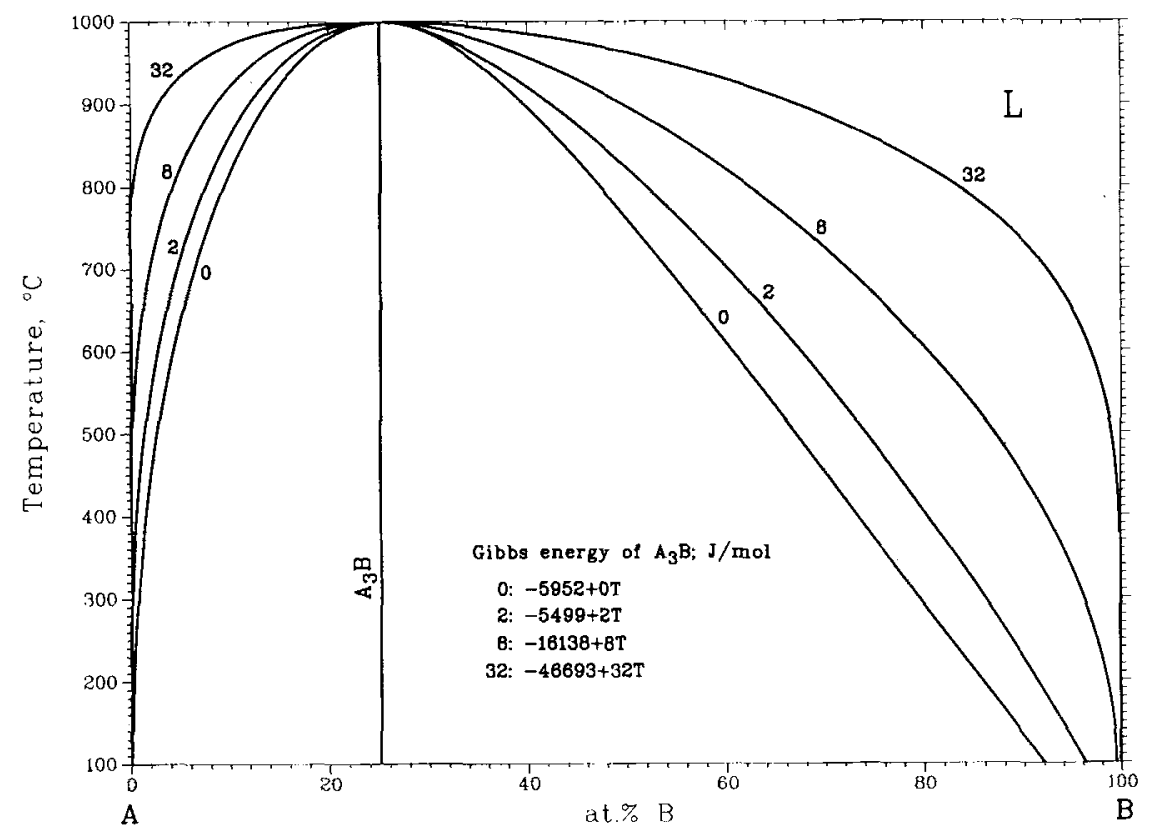

Fig. 10 Illustration of typical composition dependence of liquidus approaching 0 or 100 at.\% line. The melting point of $A_{3} B$ compound is fixed at $1000^{\circ} \mathrm{C}$, and the entropy term is varied. Mol refers to mol of atoms.

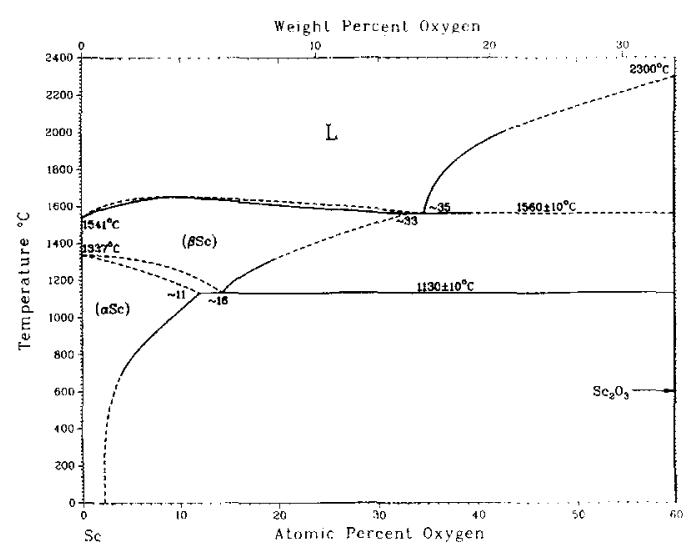

Fig. 11 Sc-O phase diagram. Redrawn from [78Mof].

The above error occurs in phase diagrams in which a eutectic or peritectic is very close to the 0 or 100 at.\% line for each element. If the proposed liquidus is drawn improperly, no room exists between the eutectic or peritectic point and the respective pure element line to allow a change in the course of the liquidus without causing the Z-type problem. To illustrate, Fig. 10 shows hypothetical trends of the liquidus calculated for an ideal liquid approaching the $B=0$ and 100 at.\% limits, respectively, for a compound $A_{3} B$ melting at $1000^{\circ} \mathrm{C}$. Although the shape changes depend on the selected constants, no abrupt change of slope is observed near the 0 or 100 at. $\% B$ compositions.

The Sc-O phase diagram (Fig. 11) [78Mof] illustrates a different problem. If the $(\mathrm{BSc}) /\left[(\beta \mathrm{Sc})+\mathrm{Sc}_{2} \mathrm{O}_{3}\right]$ solvus is extrapolated from

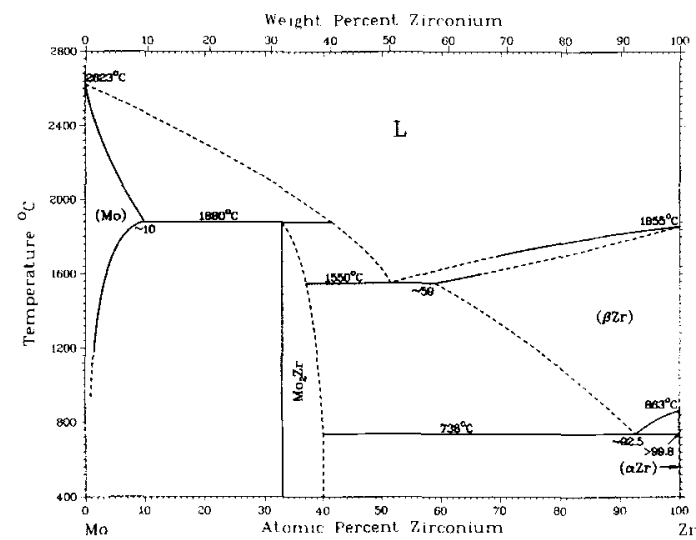

Fig. 12 Mo-Zr phase diagram. Redrawn from [76Alc].

the $\mathrm{Sc}$-rich side, it would cross the $\mathrm{L} /\left[\mathrm{L}+\mathrm{Sc}_{2} \mathrm{O}_{3}\right]$ liquidus and meet the $\mathrm{Sc}_{2} \mathrm{O}_{3}$ line below the melting point of the compound. This would make the ( $\beta S c)$-type structure at 60 at.\% $O$ more stable than the $\mathrm{Sc}_{2} \mathrm{O}_{3}$-type structure above the intersection point. This problem can be avoided if the $(\beta \mathrm{Sc}) /\left[(\boldsymbol{\beta S c})+\mathrm{Sc}_{2} \mathrm{O}_{3}\right]$ solvus turns sufficiently upward in the extrapolated range to avoid crossing the liquidus of $\mathrm{Sc}_{2} \mathrm{O}_{3}$. However, a $Y$ - or $X$-type problem then arises. Because of the apparent conflict between the $(\beta S c) /[(\beta S c)$ $\left.+\mathrm{Sc}_{2} \mathrm{O}_{3}\right]$ solvus and the $\mathrm{L}\left(\mathrm{L}+\mathrm{Sc}_{2} \mathrm{O}_{3}\right)$ liquidus, certain phase boundaries in the Sc-O phase diagram are insufficiently accurate. 


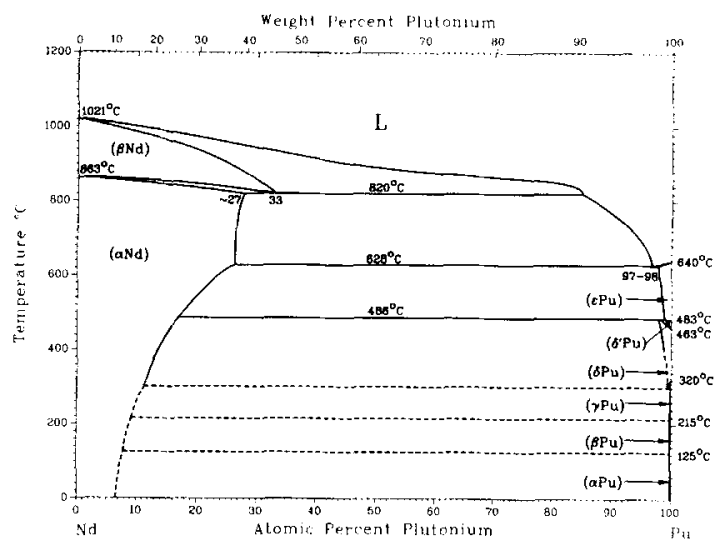

Fig. 13 Nd-Pu phase diagram. Redrawn from [78Mof].

\subsection{Problems with Phase Boundaries When the Crystal Structures May Be Known}

At first glance, the Mo-Zr phase diagram (Fig. 12) [76Alc] appears to be quite reasonable. However, with the added knowledge that the crystal structures of both (Mo) and ( $\beta Z$ r) are W-type bcc, the respective phase fields may be expected to project as a continuous solid solution (Mo, $\mathrm{BZr}$ ) in the metastable range, or as a miscibility gap between the terminal phases. Accordingly, the respective liquidus and solidus boundaries of these phases must reasonably allow for these two possibilities when extrapolated. In the former case, to avoid the $J$-type problem, the liquidus and solidus must touch at some minimum temperature as well. In the latter case, the solidus boundaries of $(\mathrm{Mo})$ and $(\beta \mathrm{Zr})$ must show retrograde solubility, smoothly touching the 0 and 100 at.\% $\mathrm{Zr}$ line, respectively, at $0 \mathrm{~K}$. Clearly, with the proposed phase diagram (Fig. 12), it is difficult to satisfy the above requirements through extrapolation of the relevant liquidus and solidus boundaries without causing a $Y$-type problem. Apparently, a significant modification is necessary.

The Nd-Pu phase diagram (Fig. 13) redrawn from [78Mof] provides another example of the $Y$ problem. When the two proposed boundaries of the $(\alpha \mathrm{Nd})+\mathrm{L}$ two-phase field are extrapolated smoothly to higher temperatures, they appear to cross at about $1100^{\circ} \mathrm{C}$, causing an obvious $T$-type problem. This problem may be alleviated by drawing a retrograde boundary on the $(\alpha \mathrm{Nd})$ side above $820^{\circ} \mathrm{C}$, so that the two boundaries cross one another at 0 at.\% Pu by smooth extrapolation. However, unless a $Y$-type problem is introduced, this hypothetical melting of $(\alpha \mathrm{Nd})$ becomes higher than that of $(\beta \mathrm{Nd})$, which is unacceptable. Accordingly, the proposed Nd-Pu phase diagram needs substantial modifications. In [90Mas], the authors proposed introducing a miscibility gap in the liquid phase to avoid the observed difficulties. Of course, the proposed diagram must be further explored experimentally.

\subsection{Problems Related to the X-Type Error}

A phase boundary with two inflection points is quite common. If elements $A$ and $B$, with substantially different melting temperatures, are immiscible in the solid state, and if the enthalpy of mixing in the liquid phase is positive and large, a miscibility gap may be expected in the liquid state above a monotectic tempera-

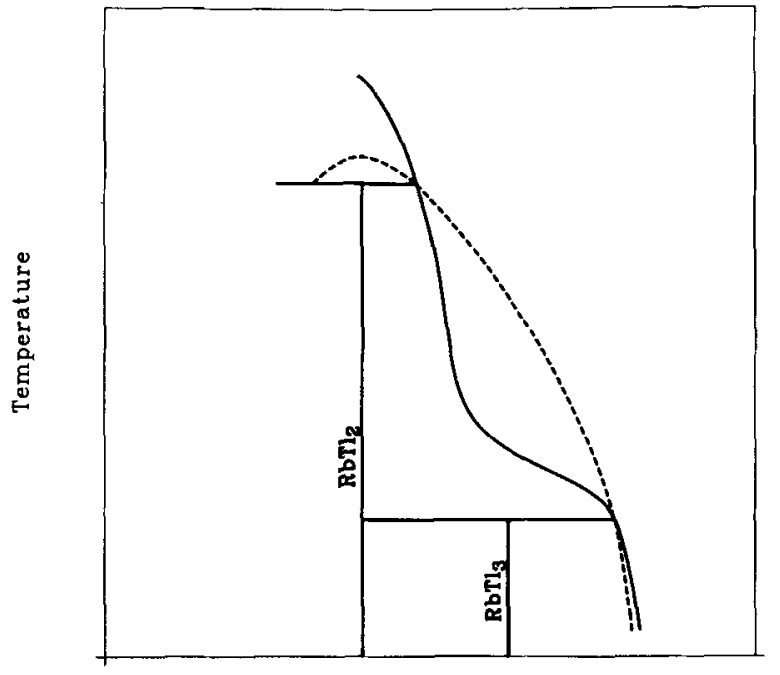

Atomic Percent TI

Fig. 14 Partial diagram of Fig. 6 indicating $X$-type problem in Fig. 1.

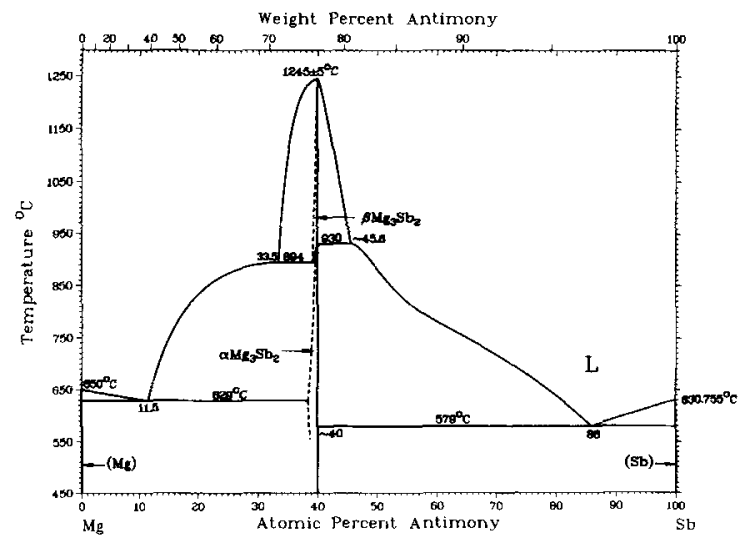

Fig. $15 \mathrm{Mg}$-Sb phase diagram. From [84Nay].

ture. (The excess entropy term is ignored here for better visualization.) By contrast, when the enthalpy of mixing is small, the phase diagram usually tends to become a simple eutectic. In between these situations, the liquidus may show inflection points, while its slope changes gradually. Although the appearance of the diagram in Fig. 14 conforms to the situation just described, if the two inflection points are located too closely to one another, as is the case for the $\mathrm{RbTl}_{2}$ liquidus in Fig. 14, an anomalous enthalpy of mixing function is required which throws doubt upon the proposed phase boundary construction (an attempt by the present authors to derive thermodynamic functions to reconcile the liquidus was unsuccessful). In this case, the liquidus deviates to the low-temperature direction from the ordinary convex form (dashed line in Fig. 14). This situation occurs if a substantial positive deviation in the enthalpy of mixing is assumed in this narrow composition range. However, it is quite unlikely that the composition dependence of any property of the liquid would deviate 


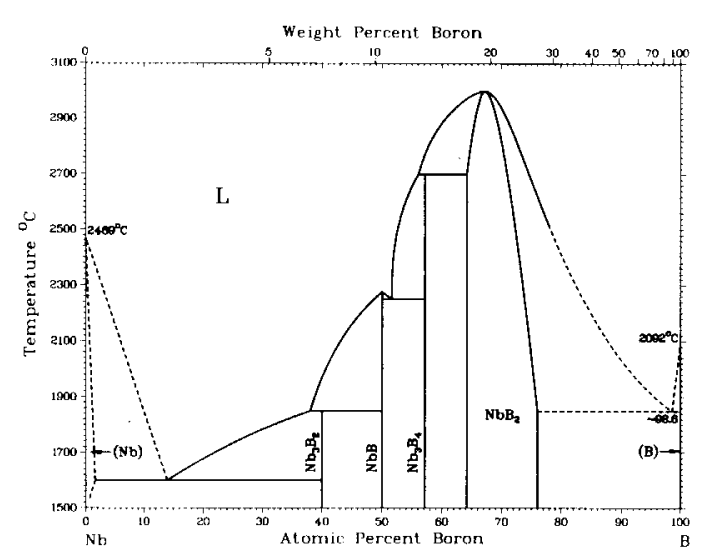

Fig. $16 \mathrm{Nb}-\mathrm{B}$ phase diagram. Redrawn from [65Ell].

from the general trend in a narrow composition range, particularly if this is to be a positive deviation. Thus, we again reach the conclusion that the form of the $\mathrm{RbTl}_{2}$ liquidus is practically impossible to reconcile thermodynamically.

The $\mathrm{Mg}$-Sb phase diagram [84Nay] (Fig. 15) shows the $X$-type problem occurring in the metastable range. When the liquidus boundaries of $\alpha \mathrm{Mg}_{3} \mathrm{Sb}_{2}$ are extrapolated to higher temperatures, the metastable melting point of $\alpha \mathrm{Mg}_{3} \mathrm{Sb}_{2}$ is estimated to be at $-900^{\circ} \mathrm{C}$ from the $\mathrm{Mg}$-rich liquidus and $-950^{\circ} \mathrm{C}$ from the $\mathrm{Sb}$-rich liquidus. Because there must be only one projected melting point, the extrapolation of the $\mathrm{Mg}$-rich liquidus above the peritectic temperature must be drawn to meet the extrapolation from the Sbrich side at the composition of $\mathrm{Mg}_{3} \mathrm{Sb}_{2}$. However, an $X$-type problem then becomes unavoidable. Most likely, the form of the liquidus on the $\mathrm{Mg}$-rich side in the range of 33 at.\% Sb should not be as flat as proposed.

A similar problem occurs in the Nb-B phase diagram (Fig. 16) [65Ell], in which the $\left.L / L+\mathrm{NbB}_{2}\right)$ liquidus is concave near the eutectic. Because the liquidus must change the sign of curvature when extrapolated below the eutectic temperature (to avoid crossing the B $=100$ at. \% line), a very abrupt change of slope is predicted if the eutectic is as close to the pure $B$ side as proposed.

An interesting point is illustrated in Fig. 17. Here, we suspect that in order to avoid an assumed $K$-type problem presented by a discontinuous-appearing boundary, the $\mathrm{Cr}_{3} \mathrm{O}$ s phase boundary in Fig. 17 [90Ven1] was drawn with a rounded maximum touching the invariant line at $1540^{\circ} \mathrm{C}$. However, if the $\mathrm{Cr}_{3} \mathrm{O}$ s phase forms through a peritectoid reaction $(\mathrm{Cr})+\sigma \leftrightarrow \mathrm{Cr}_{3} \mathrm{Os}$, the left and the right side boundaries of the $\mathrm{Cr}_{3} \mathrm{Os}$ phase field are actually separate lines governed by the respective thermodynamics of $\mathrm{Cr}_{3} \mathrm{Os}$ with respect to $(\mathrm{Cr})$ and $\sigma$. Therefore, the top of the $\mathrm{Cr}_{3} \mathrm{Os}-$ phase field is not a maximum in a continuous boundary, but merely the intersection of two boundaries, which occurs precisely on the horizontal invariant temperature line. Thus, the slope at the contact point need not be horizontal. To avoid the $N$-type problem in each of the boundaries, strong inflections must be assumed to exist in the extrapolations of the presently proposed phase boundaries on both sides of $\mathrm{Cr}_{3} \mathrm{O}$ s near the peritectoid temperature, creating the $X$-type problem in each segment. Most likely, the actual top of the $\mathrm{Cr}_{3} \mathrm{O}$ s phase field involves the usual pointed shape.

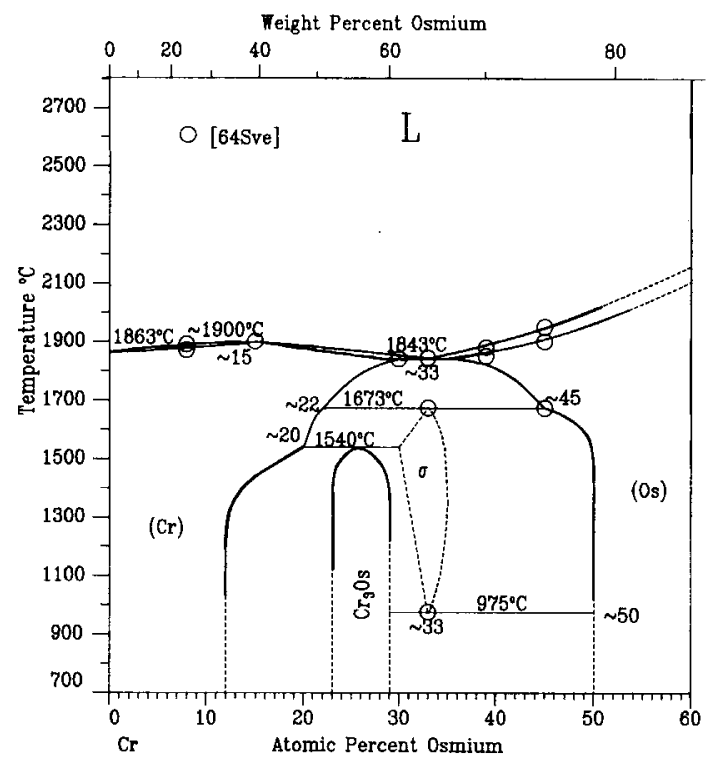

Fig. 17 Partial Cr-Os phase diagram. From [90Ven1].

\section{Less Obvious Phase Diagram Situations}

\subsection{Consistency of Initial Slopes in Relation to the van't Hoff Equation}

If the slopes of liquidus and solidus of a terminal solid solution are wide apart (ie., a nearly horizontal liquidus and a nearly vertical solidus), or if their slopes are almost the same, the situation should be examined further for possible difficulties. The well-known van't Hoff relationship for the initial slopes of the liquidus and solidus

$(d X / d T)_{\text {solidus }}-(d X / d T)_{\text {liquidus }}=\Delta_{\text {fus }} H / R T_{\mathrm{m}} 2$

where $X$ is the mole fraction solute, requires that the difference of the inverse values of the initial slopes (not angles) must be apart by a certain amount determined by the enthalpy of fusion, $\Delta_{\mathrm{fus}} \mathrm{H}$, and the melting point, $T_{\mathrm{m}}(\mathrm{K})$, of the pure element itself. Because the differences in the $\Delta_{\mathrm{fus}} \mathrm{H}$ and $T_{\mathrm{m}}$ of metallic elements are not very large, the initial slopes of liquidus and solidus must be "properly" apart. Table 1 shows the calculated expected separation between the initial liquidus and solidus in terms of the temperature drop of the liquidus, assuming $d X / d T$ for the solid phase is 0 (vertical drop) and using the $\Delta_{\text {fus }} H$ values as published in [83Cha].* Thus, the numbers in Table 1 indicate the initial slope of the liquidus for all systems based on a given element, expressed in terms of the temperature drop per $100 \%$ solute when there is no solubility in the solid phase. To illustrate, the calculated initial slopes for nearly all elements have been drawn as extended straight lines in Fig. 18(a) to 100 at.\% solute (some elements with allotropic transformations are not shown). Surprisingly, the lines for all metallic elements (first group) almost always converge to a fixed point $\left(130 \pm 10\right.$ at. $\left.\%,-500 \pm 50^{\circ} \mathrm{C}\right)$. Some metallic elements

*Some are from [73Hul] as mentioned below. 
did not follow this interesting empirical trend, and possible causes of these exceptions were considered, assuming that the empirically observed relationship has some general universal meaning for all elements. First, the calculated slopes for most of the rare earth elements are rather high (Table 1), which is inconsistent with the general trend. This anomaly may be explained, however, by the occurrence of allotropic transformations in many rare earth elements. For the low-temperature allotropic forms, the calculated slope becomes less steep, satisfying the general rule. As a matter of fact, rare earth elements having no allotropic transformations (Ho, Tm, Lu) are not exceptions in Fig. 18(a). Similarly, some metallic elements ( $\mathrm{Fe}, \mathrm{Mn}, \mathrm{Ca}, \mathrm{Sr}$, etc.) appear to show slopes that are slightly too steep to agree with the general rule (Fig. 18a). Here again, however, these elements have allotropes and the low temperature allotropic forms satisfy the rule. Fig. 18(b) shows some examples of this, indicating that the present empirical rule holds true if low-temperature allotropes are considered.

Subsequently, further refinements to our general plots in Fig. 18(a) became possible. For example, according to our preliminary calculations using $\Delta_{\text {fus }} H$ values given in [83Cha], the slopes for $\mathrm{W}, \mathrm{Mo}$, and $\mathrm{Hf}$ appeared to be too flat (as if converging to about 160 at.\% instead of 130 at. \%), and the slope for Ba was too steep (as if converging to $\sim 105$ at.\%). This anomaly could be eliminated in the final plot for these elements, Fig. 18(a), by using slopes calculated with the $\Delta_{\text {fus }} H$ given in [73Hul]. (For Mo, the lowest value within the error limit was plotted.) With this modification, no apparent inconsistency remains for these elements. Finally, some actinides $\mathrm{Pa}, \mathrm{Th}, \mathrm{U}$, and $\mathrm{Pu}$, including the allotropic transformations) still showed a deviation from the rule. Because of the success in Fig. 18, it is tempting to suggest that the deviation might be due to currently inaccurate $\Delta_{\text {fus }} H$ or enthalpy of transformation data as given in [83Cha]. Table 2 gives enthalpies of fusion calculated from an assumed conformity to the observed behavior in Fig. 18(a), using the fixed point of convergence at $130 \mathrm{at} . \%$ and $-500^{\circ} \mathrm{C}$ for the metallic elements having no allotropic transformations. Considering the scatter among the reported values, if the data in Table 2 are compared with the data on enthalpies reported by [83Cha], [87Alc], [90Din], and [73Hul], the calculated values agree surprisingly well, with only a few exceptions (third group, as discussed below).

Nonmetallic and semimetallic elements (B, Si, Ge, Sb, Te, Se, Bi, $\mathrm{Sn})$ appear to form a second group, with a converging point roughly at 260 at.\% (twice that of the first metallic group) and $-500^{\circ} \mathrm{C}$. Athird group including elements $\mathrm{Al}, \mathrm{Zn}, \mathrm{Cd}, \mathrm{Hg}$, and $\mathrm{Ga}$

Table 1 Initial Slope of Liquidus When the Solidus is Vertical

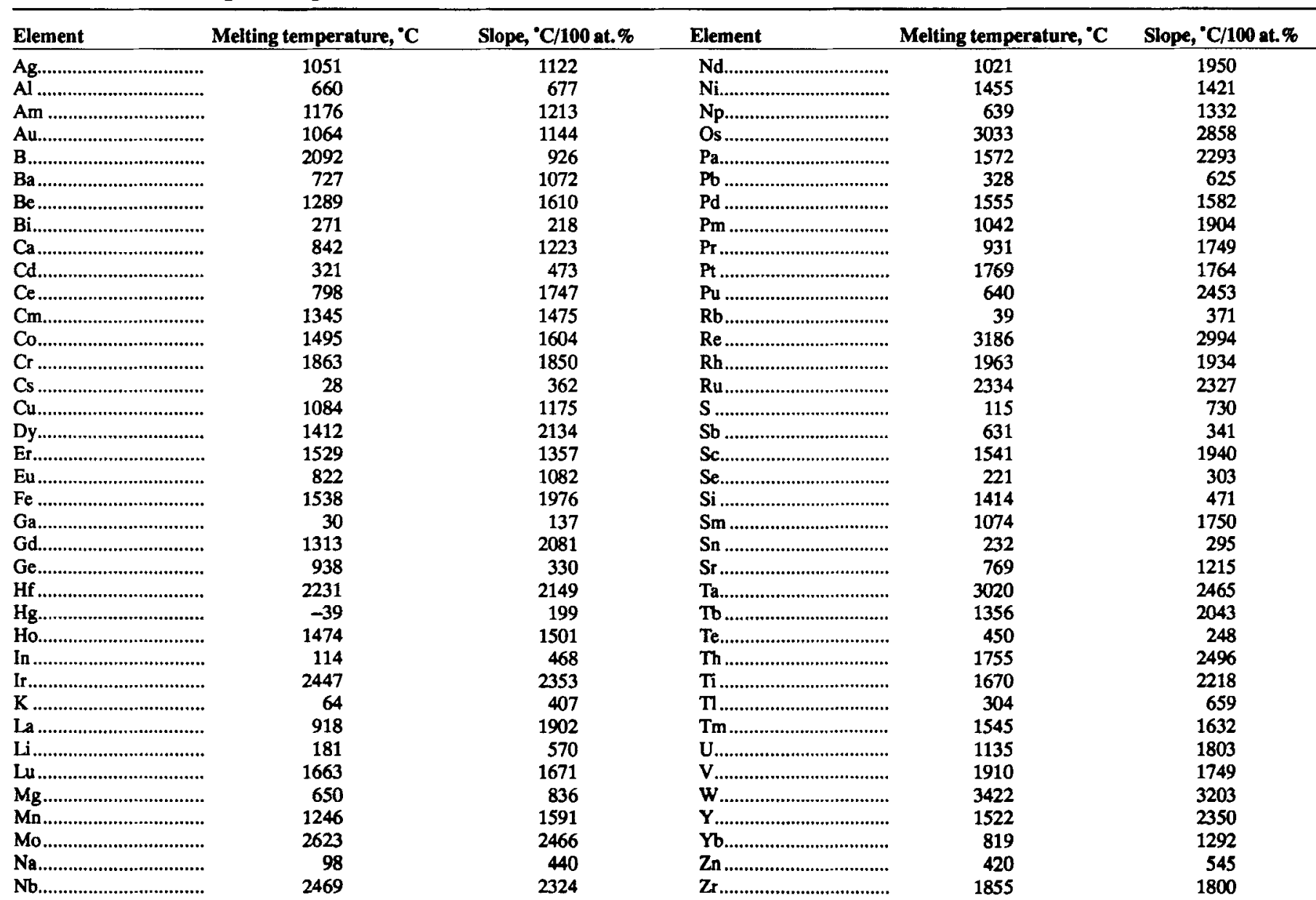

Note: The slope number is temperature drop, ${ }^{\circ} \mathrm{C} / 100$ at.\% secondary element, calculated from the enthalpy of fusion given by [83Cha]. 
(neighbors in the periodic table) appears to converge to a point between those of the first and the second groups.

We conclude from the above observations that when the solid solubility is negligible, the initial slope of the liquidus of a metallic element (having no allotropic transformation in principle) must be drawn to pass through a point at 130 at. $\%$ and $-500^{\circ} \mathrm{C}$. As a general rule, therefore, when checking a proposed phase diagram, the initial liquidus slope is expected to point at somewhere between $0 \mathrm{~K}$ and $0^{\circ} \mathrm{C}$ at the solute side of the phase diagram, especially if little solid solubility exists. This temperature is somewhat higher for elements with relatively high melting points, but because the slopes are steeper, the associated errors are not a problem for the purpose of an initial rough check. On the other hand,

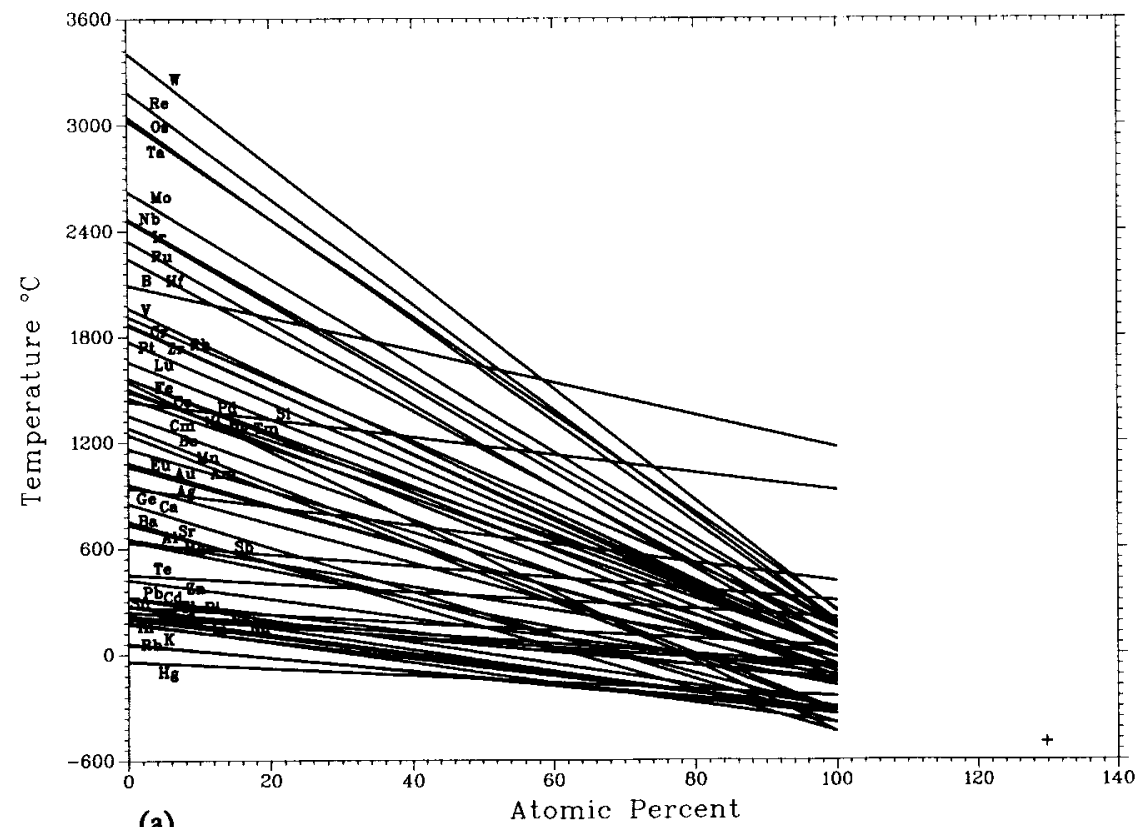

(a)

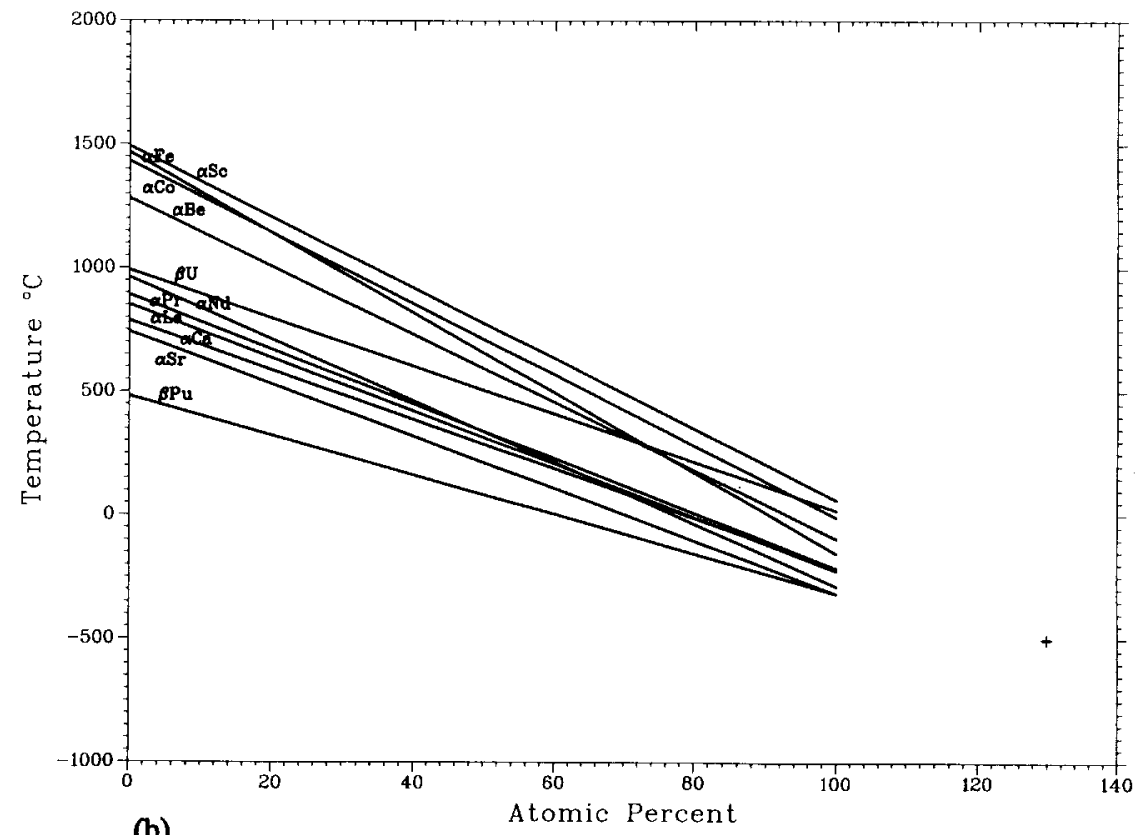

(b)

Fig. 18 (a) Illustration of convergence of the initial slope to $\sim 130$ at. $\%$ and $-500^{\circ} \mathrm{C}$. Some rare earth elements are not included because of apparent deviation to the low temperature side caused by omission of allotropic transformations. (b) Supplementary figure for low-temperature allotropes. 
Table 2 Enthalpy of Fusion of Metallic Elements with No Allotropes (J/mol)

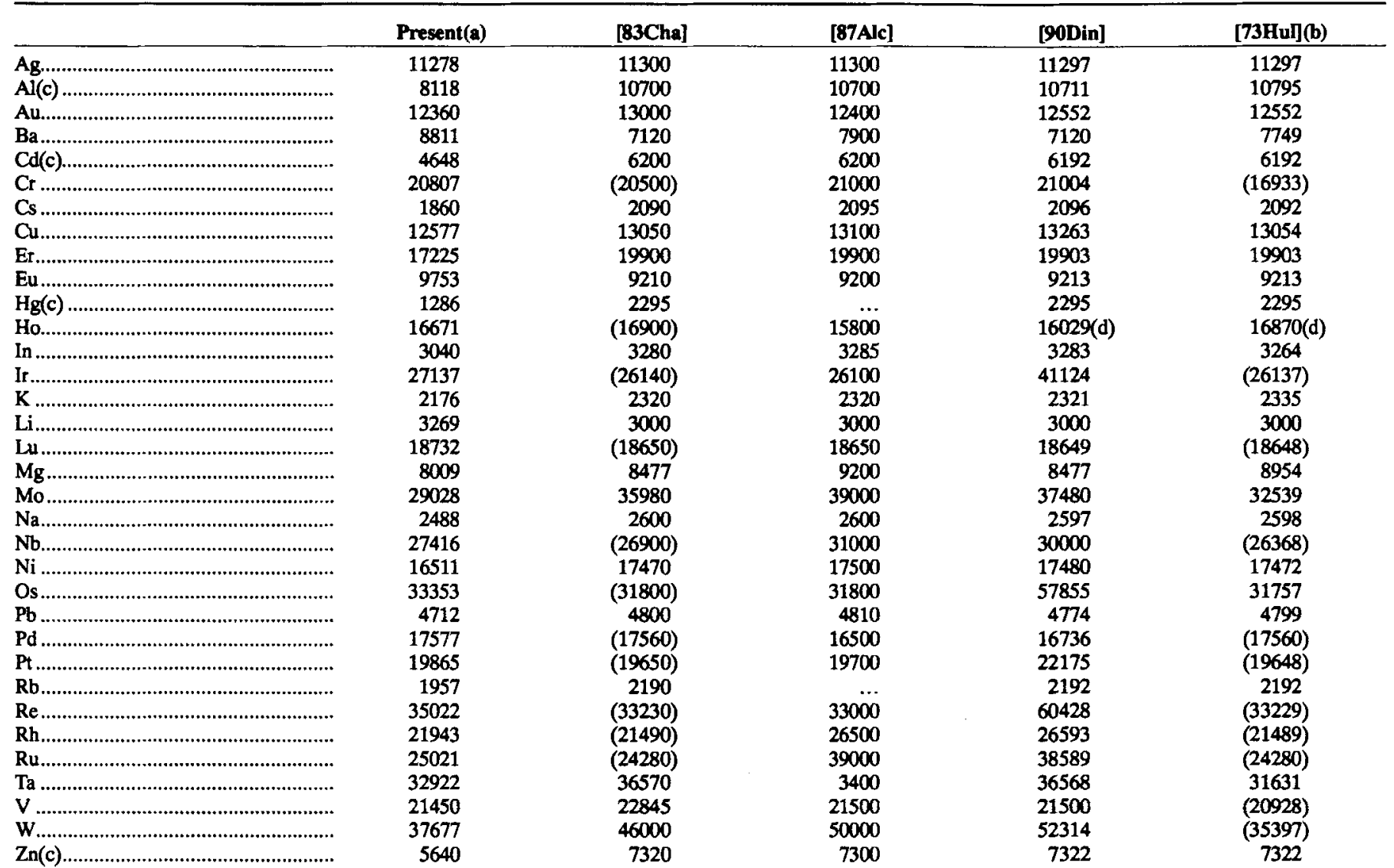

Note: Numbers in parentheses are tentative. (a) Calculated from the 130 at. $\%,-500{ }^{\circ} \mathrm{C}$ point (see text). (b) $4.184 \times$ original value in cal/mol. (c) Element showing substantial deviation. (d) Nonexistent allotropic transformation is included.

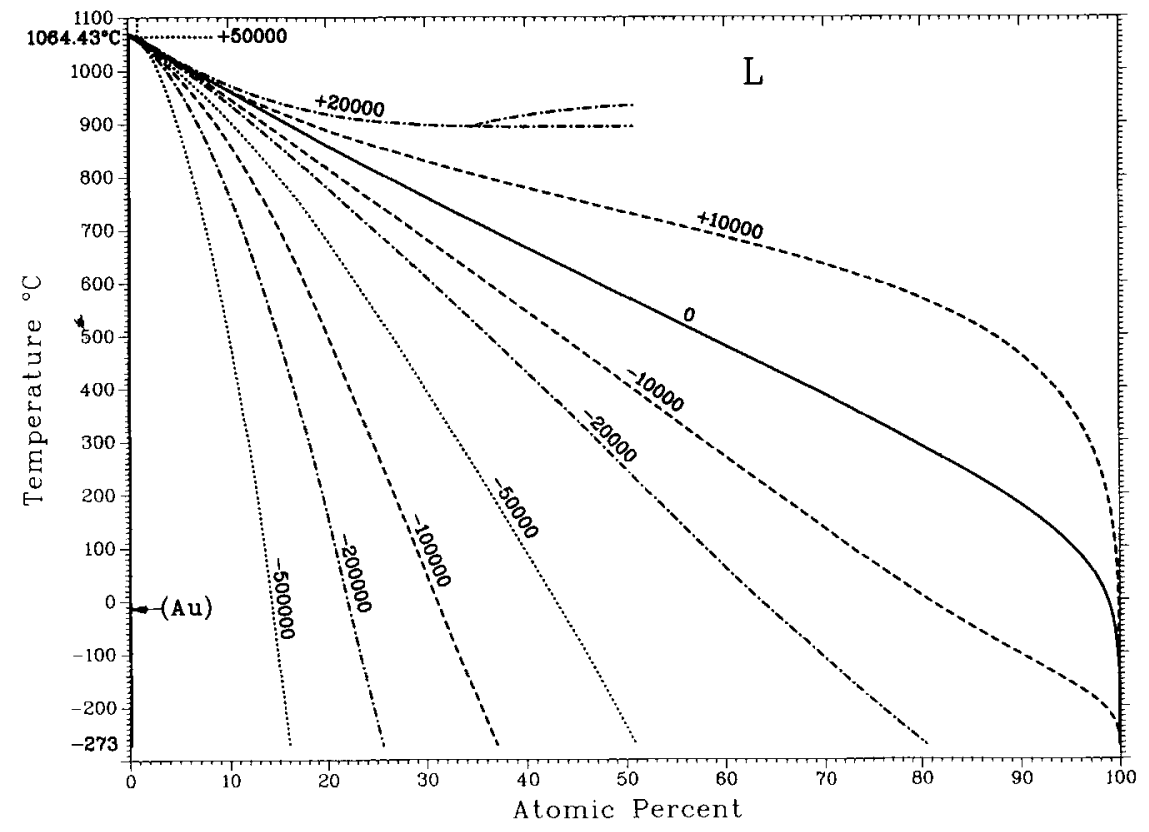

Fig. 19 Liquidus of (Au) with no solid solubility. Liquid is a regular solution. Numbers represent interaction parameters (J/mol). 
the converging temperature point is somewhat lower for elements exhibiting allotropic changes. It appears fairly safe to conclude from our overwhelming data that if the initial slope of the liquidus is substantially steeper (or flatter) than that expected from the empirical rule discussed above, the phase diagram details in the dilute region may be suspect. Naturally, it is rather intriguing to gain some theoretical insight into the observed behavior in the dilute alloy regions.

Further considerations indicate that, in addition to the direction of the initial slope, the subsequent actual trend of the liquidus is also subject to additional restrictions, because the magnitude of the excess Gibbs energy of mixing of a liquid phase cannot be expected to take unrealistic values. Thus, as an example, Fig. 19 shows the influence of the magnitude of excess Gibbs energy on the form of the liquidus boundary for the case of Au-based binary systems. The (Au) solid phase is assumed to involve no solid solubility. The lattice stability parameter of $\mathrm{Au}$ is the same as that used for Fig. 18(a). Liquidus curves shown in Fig. 19 have been calculated assuming a regular solution behavior for the liquid phase, i.e., $G^{\operatorname{ex}}(\mathrm{L})=\Omega X(1-X)$, taking $\Omega$ to be $50000,20000,10000$, $0,-10000,-20000,-50000,-100000,-200000$, and $-500000 \mathrm{~J} / \mathrm{mol}$. ( $X$ is atomic fraction of solute.) When $\Omega$ is large and positive (>19 $100 \mathrm{~J} / \mathrm{mol}$ ), a miscibility gap develops in the liquid phase. Therefore, only a part of the liquidus is shown for $\Omega$

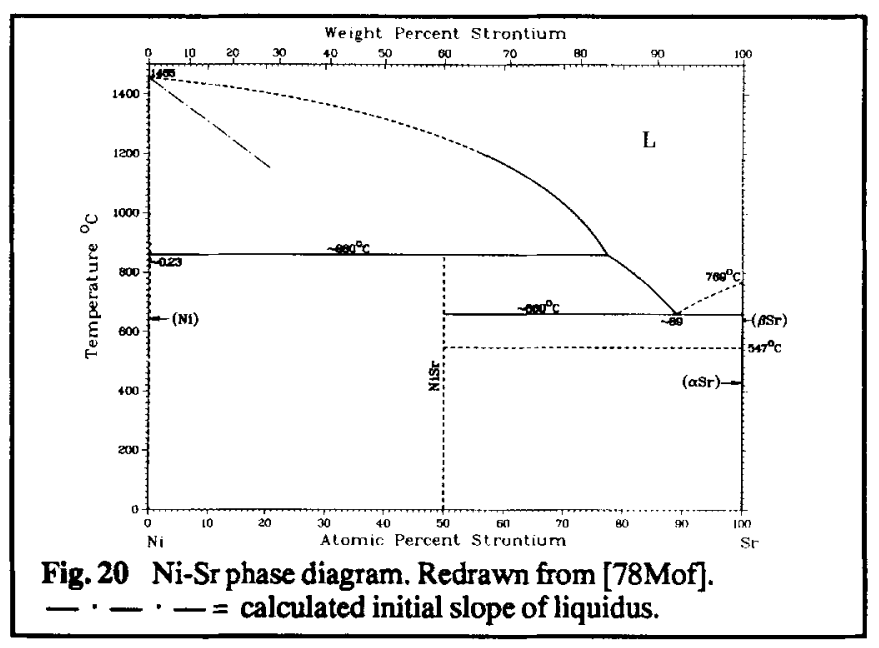

Fig. 20 Ni-Sr phase diagram. Redrawn from [78Mof]

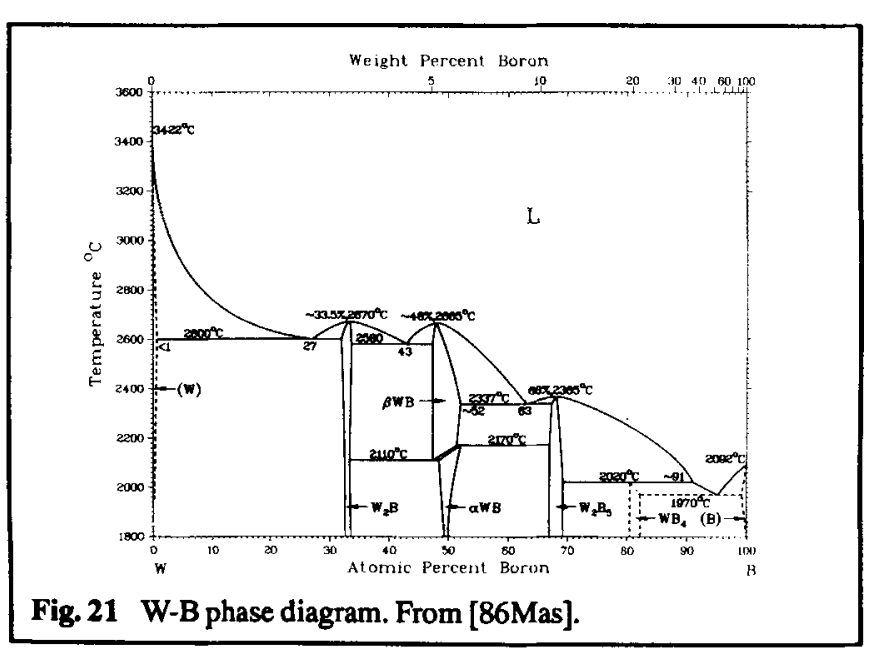

$=50000$ and $20000 \mathrm{~J} / \mathrm{mol}$. Although Fig. 19 shows a calculated trend of the liquidus for $\Omega$ down to $-500000 \mathrm{~J} / \mathrm{mol}$, this value is unusually low for any metallic system. Considering the values of $\Omega$ usually quoted in literature (e.g., the Monograph Series for binary alloy phase diagrams published by ASM), it may be a fair generalization to accept that $-100000 \mathrm{~J} / \mathrm{mol}$ is an exceptionally low value for $\Omega$. Therefore, when the (Au) phase involves little or no solid solubility, the liquidus trend in the corresponding phase diagram must always fall roughly in the range between the curves in Fig. 19 calculated for $\Omega$ between 10000 and $-100000 \mathrm{~J} / \mathrm{mol}$ or develop a miscibility gap. Any liquidus in Au-based systems showing significant deviation from this typical behavior requires careful scrutiny. In Fig. 19, the composition dependence and temperature dependence of $\Omega$ was not considered. However, any added composition dependence would not change the form of the curve very much, because small changes in $\Omega$ do not alter the course of the liquidus significantly, especially when $X$ is small as shown in the Figure. Finally, the influence of the temperature dependence of $\Omega$ (primarily the excess entropy term) should also be small, because even for a very high value of $100 \mathrm{~J} / \mathrm{mol}$, the deviation from the mean value (i.e., when $T S$ ex is replaced by $\overline{T S}$ ex) is only some $5000 \mathrm{~J} / \mathrm{mol}$ per $100^{\circ} \mathrm{C}$. If we add the above considerations to the empirical rule concerning the initial slopes of the liquidus curves of most metallic elements, which have been shown in Fig. 18(a) to conform to a consistent general pattern, the general liquidus trends as depicted in Fig. 19 are presumably valid for the majority of metallic alloy systems.

\subsection{Observed Problems with Initial Slopes}

The generalizations discussed above and the accompanying usefulness of the van't Hoff relationship are employed in a few specific examples. Because the $d X / d T$ of $(\mathrm{Ni})$ solidus in the $\mathrm{Ni}-\mathrm{Sr}$ system (Fig. 20) [78Mof] is nearly 0 , the liquidus slope must be steeper than that indicated $\left(1421^{\circ} \mathrm{C} / 100 \%\right.$ temperature drop indicated from Table 1). This appears to conflict with the liquidus trend projected at higher contents of Sr. It is very likely that a miscibility gap exists in the liquid state, as in the rather similar $\mathrm{Ni}-\mathrm{Ba}$ system (see [90Mas]).

The initial slope of the (W) liquidus in the W-B system (Fig. 21) [86Mas] (redrawn from [78Mof] which is based on [69Rud]) provides an extreme example in the opposite direction. Here, the

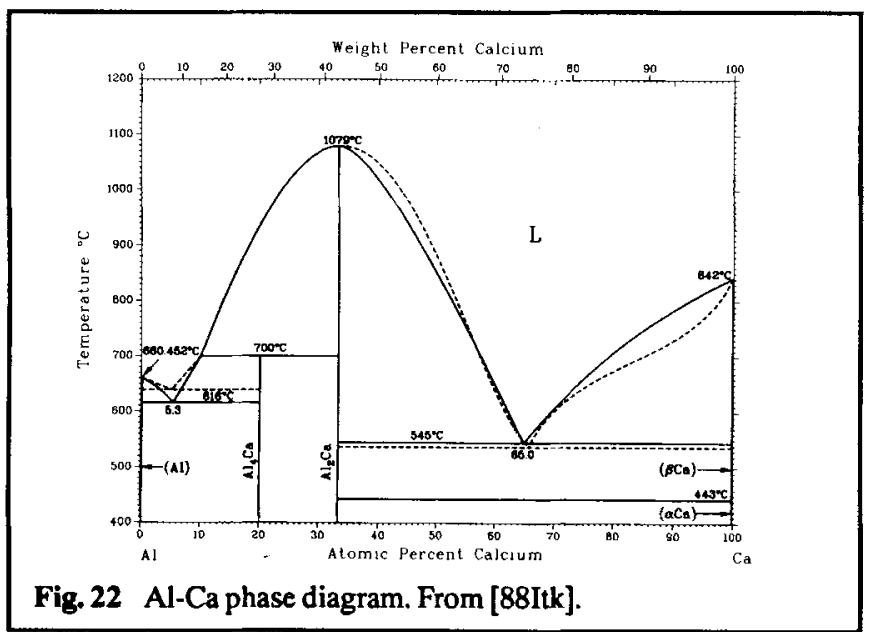




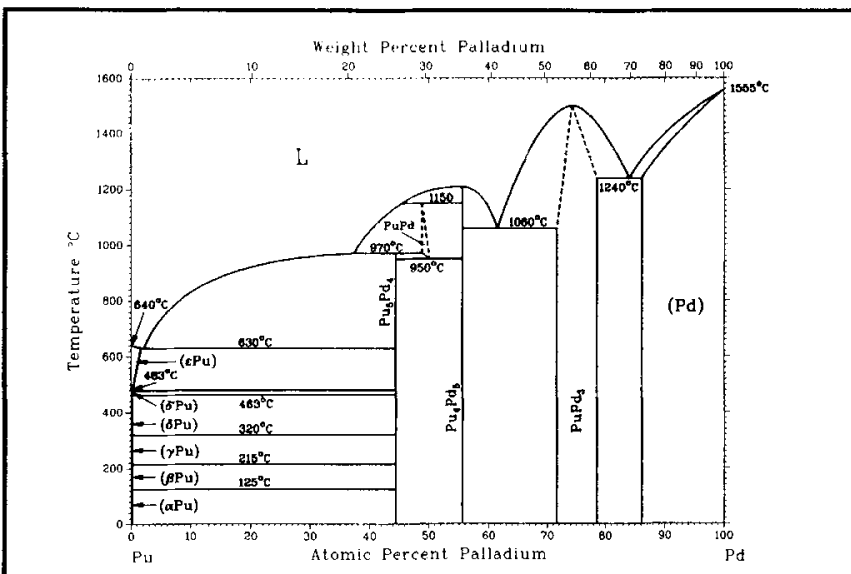

Fig. 23 Pu-Pd phase diagram. Redrawn from [78Mof].

Table 3 Cu-Lu Thermodynamic Properties [88Sub]

Lattice stability parameters for $\mathrm{Cu}$

$G^{0}(\mathrm{Cu}, \mathrm{L})=0$

$G^{0}(\mathrm{Cu}, \mathrm{fcc})=-13054+9.613 T$

Lattice stability parameters for $\mathrm{Lu}$

$G^{0}(\mathrm{Lu}, \mathrm{L})=0$

$G^{0}(\mathrm{Lu}, \mathrm{cph})=-18650+9.633 T$

Integral molar Gibbs energies

$G(L)=X(1-X)(-79178+46599 X)+R T[X \ln X+(1-X) \ln (1-X)]$

$G\left(\mathrm{Cu}_{5} \mathrm{Lu}\right)=-24862+7.75 \mathrm{~T}$

$G\left(C u_{g} L u_{2}\right)=-28462+9.72 T$

$G\left(\mathrm{Cu}_{2} \mathrm{Lu}\right)=-15194+4.47 T$

$G($ CuLu $)=-29290+4.64 T$

Note: Gibbs energies are expressed in $\mathrm{J} / \mathrm{mol}$, and temperatures are in $\mathrm{K} . X$ is the atomic fraction of $\mathrm{Lu}$. Mol refers to the atom as the elementary entity.

slope is apparently too steep. (It was not so steep in the diagrams of [69Rud] and [78Mof], but there was an unusual change of slope at $\sim 1$ at. \% B.)

The empirical rule derived above is also useful for a quick examination of calculated phase diagrams for the systems projected to have terminal phases with negligible solid solubility. In the AlCa phase diagram (Fig. 22) [88Itk], the initial slopes of calculated $(\mathrm{Al})$ and $(\mathrm{BCa})$ liquidus lines (dashed lines) are pointing very much above $0{ }^{\circ} \mathrm{C}$ and below $0 \mathrm{~K}$, respectively, on the respective sides of the phase diagram. Therefore, errors in calculating or drawing are suspected.

In the Pd-Pu phase diagram (Fig. 23) [78Mof], the reported slopes of liquidus and solidus of (Pd) are approximately -15 and -20 ${ }^{\circ} \mathrm{C} / \mathrm{at} . \%$, respectively. If the liquidus is correct, the solidus slope should be about $-290^{\circ} \mathrm{C} /$ at. $\%$. If the solidus is correct, the liquidus slope should be $-9^{\circ} \mathrm{C} /$ at. \%. Hence, the liquidus and solidus of (Pd) in Fig. 23 are apparently too close to one another.

Because the enthalpy of boiling of an element is generally very much higher than the enthalpy of fusion, the initial opening of the $(G+L)$ two-phase field should be much wider compared with that of the $(\mathrm{L}+\mathrm{s})$ two-phase field. As an example, the schematic drawing of the gas phase in the Ge-C system (Fig. 24) [84Ole], which

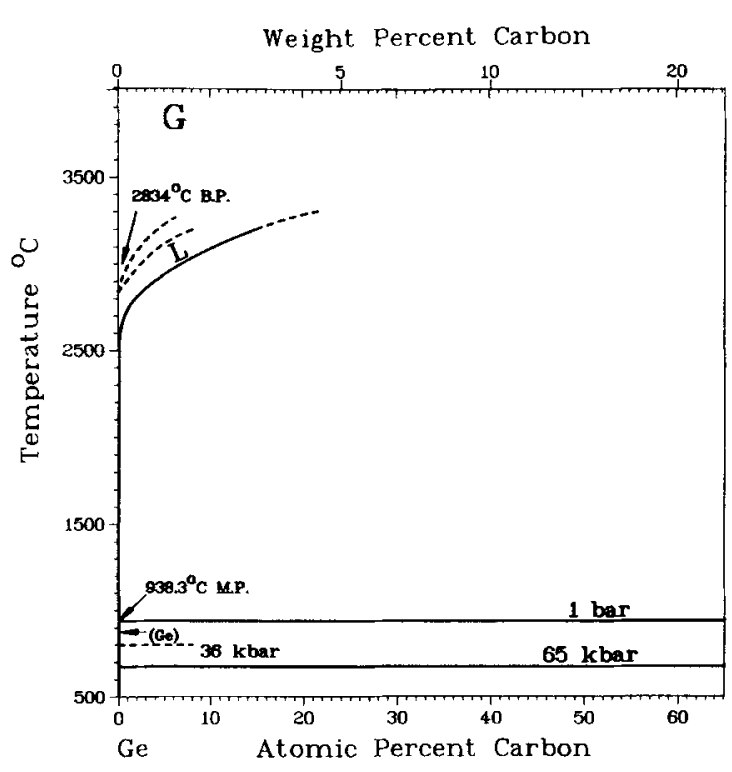

Fig. 24 Partial Ge-C phase diagram. From [84Ole].

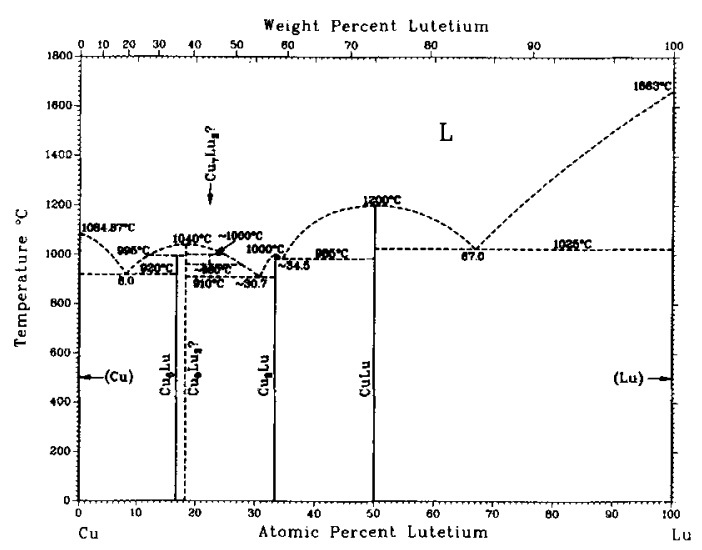

Fig. $25 \mathrm{Cu}$-Lu phase diagram. From [88Sub].

was presumably added to indicate the boiling of $\mathrm{Ge}$, neglects this point and is unlikely. The two-phase field between gas and liquid must converge into one point at the boiling point on each side of a phase diagram, a point often neglected when the gas phase is included. (See also "5. Suspicious or Erroneous Diagrams with Seemingly Acceptable Appearance".)

\subsection{Mixed Degrees of Curvature in the Liqui- dus if More than Two Compounds are Present}

A comparison of the respective liquidus curvatures at the melting points of several intermetallic compounds can reveal thermodynamically unlikely situations. As may be expected, if the melting point of a compound is fixed, an increasingly negative enthalpy of formation assumed for that compound in a thermodynamic modeling calculation probably leads to a relatively slow fall of the liquidus on both sides. Thus, in a situation when a number of compounds follow one another in a phase diagram, a compound with a pointed liquidus is likely to be less stable in comparison with 


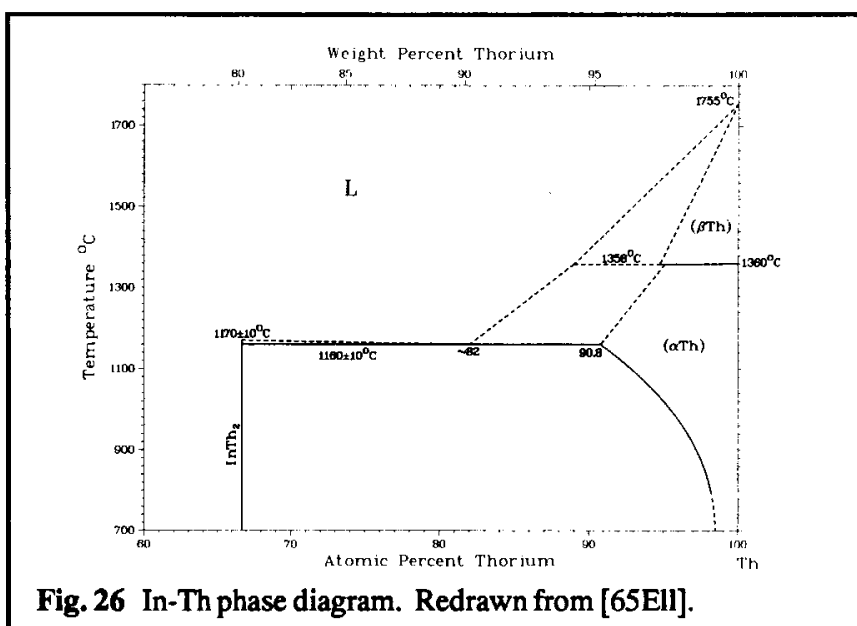

ones having a flat liquidus. This situation is tested in the $\mathrm{Cu}-\mathrm{Lu}$ phase diagram (Fig. 25) [88Sub]. Here, the liquidus of $\mathrm{Cu}_{2} \mathrm{Lu}$ is proposed to be relatively steep, whereas that of $\mathrm{CuLu}$ is relatively flat. However, the proposed thermodynamics do not bear this out. The Gibbs energies of the $\mathrm{Cu}-\mathrm{Lu}$ compounds proposed by [88Sub] are given in Table 3. At $T=0 \mathrm{~K}$, the Gibbs energy (enthalpy) of $\mathrm{Cu}_{2} \mathrm{Lu}(-15194 \mathrm{~J} / \mathrm{mol})^{*}$ is substantially higher than those of the neighboring compounds $\left(-28462 \mathrm{~J} / \mathrm{mol}\right.$ for $\mathrm{Cu}_{9} \mathrm{Lu}_{2}$ and $-29290 \mathrm{~J} / \mathrm{mol}$ for $\mathrm{CuLu}$ ). Therefore, in this thermodynamic model, $\mathrm{Cu}_{2} \mathrm{Lu}$ is definitely unstable at $0 \mathrm{~K}$ and is predicted to be unstable up to $4550^{\circ} \mathrm{C}$ with respect to the two neighboring compounds. At this temperature, the liquid is more stable than all the solid phases; therefore, $\mathrm{Cu}_{2} \mathrm{Lu}$ cannot exist at any temperature. However, the existence of $\mathrm{Cu}_{2} \mathrm{Lu}$ is observed experimentally as an equilibrium phase at low temperatures, and so the Gibbs energy model for the proposed compounds must be adjusted to allow for this.

Figure 10 already illustrated that the Gibbs energy at $T=0 \mathrm{~K}$ (enthalpy term of the Gibbs energy) is related to the resulting sharpness of the liquidus curvature. If the sharpness of the liquidus curves at the congruent points of several intermetallic compounds varies significantly, the compound with the sharpest liquidus will be the least stable at low temperatures, and may be altogether unstable in an equilibrium diagram.

As a general rule, investigate further if the curvature of the liquidus of a given compound is very sharp, or very flat, in comparison with those of the neighboring compounds, even if the thermodynamical data are not available. For example, the proposed liquidus of $\mathrm{InTh}_{2}$ shown in [65Ell] (Fig. 26) is strikingly flat. $\mathrm{InTh}_{2}$ would be extremely stable at low temperatures and no other compounds are likely to coexist. However, a more recent study of the In-Th phase diagram [84Pal] showed that the very flat peak is not real (see [90Mas]).

\subsection{Miscibility Gap Away from the Center of a Phase Dlagram}

An interesting point may be made in connection with proposed miscibility gaps. Figure 27 shows the proposed V-Sn phase diagram [81Smi], in which existence of a liquid immiscibility is pos-

* Mol in this article refers to mol of atoms, i.e., g-atom.

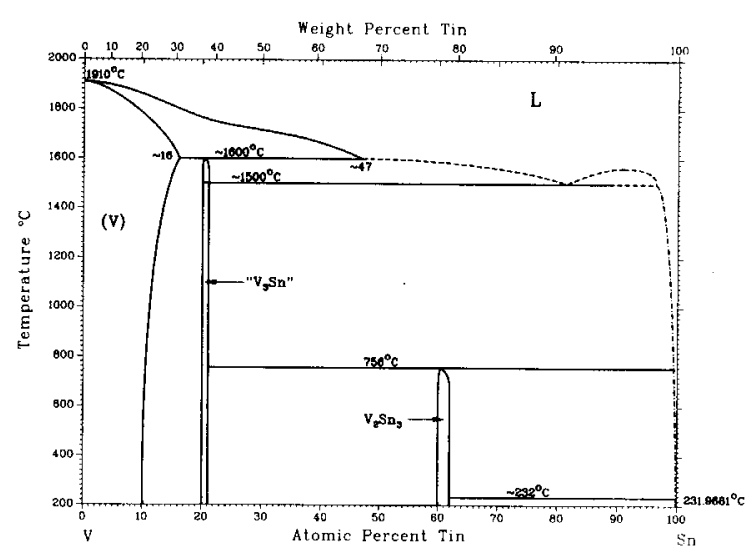

Fig. 27 V-Sn phase diagram. From [81Smi].

tulated with a critical point at about 90 at.\% Sn. Generally, immiscibility in the liquid or solid state occurs most frequently near the equiatomic composition. (When there is a molecular liquid phase, it occurs at a composition somewhere in the middle between the molecular liquid phase and an element.) This situation is quite understandable, if the interaction between unlike atoms is of the Bragg-Williams type, or if a deviation from this general type is realistic. A miscibility gap can occur near an edge of a phase diagram if the composition dependence of the excess Gibbs energy shows a change from very large negative values to very large positive values, which is unlikely. Alternatively, the composition dependence would have to be positive at all compositions, except one showing a very high-order composition dependence, which is also unlikely. Therefore, miscibility gaps indicated near extreme ends of phase diagrams merit particular scrutiny.

\subsection{Melting of a Stoichiometric Compound off the Stolchiometric Composition}

When a phase designated as a stoichiometric compound $A_{m} B_{n}$ has a substantial solid solubility range, the observed highest melting temperature of the compound need not coincide with the stoichiometric composition. This is because the Gibbs energy curves of the liquid phase and the $A_{m} B_{n}$ compound may touch at a composition considerably displaced from the stoichiometric composition, even though the actual minimum of the Gibbs energy of the $A_{m} B_{n}$ compound is at the exact composition $A_{m} B_{n}$. However, in the majority of the binary phase diagrams [90Mas], the deviation of the composition of the observed congruent melting point from the ideal stoichiometry is at most 1 to 2 at.\%. Hence, if the congruent melting point of a phase obtained by projecting the liquidus into the metastable region deviates substantially from stoichiometry, a closer look at the phase diagram may be warranted. For example, the metastable melting of $\mathrm{Al}_{3} \mathrm{Ni}_{2}$ in the $\mathrm{Al}-\mathrm{Ni}$ phase diagram (Fig. 28) [90Sin] appears to occur at 45 to 50 at.\% $\mathrm{Al}$ when the liquidus and the solidus of $\mathrm{Al}_{3} \mathrm{Ni}_{2}$ are extrapolated toward higher temperatures above the peritectic formation temperature of $1133^{\circ} \mathrm{C}$ (dashed lines in Fig. 28). Because the crystal structure of $\mathrm{Al}_{3} \mathrm{Ni}_{2}$ is a prototype, the (metastable) melting of this phase is expected to occur at a composition close to its stoichiometry. It seems that the phase boundaries of $\mathrm{Al}_{3} \mathrm{Ni}_{2}$ appear to require some modifications. A very minor alteration (e.g., move the 
composition of $\mathrm{Al}_{3} \mathrm{Ni}_{2}$ at $1133^{\circ} \mathrm{C}$ to $\sim 38$ at. $\% \mathrm{Ni}$ ) would alleviate the difficulty of the off-stoichiometric melting of $\mathrm{Al}_{3} \mathrm{Ni}_{2}$.

\section{Unusual Phase Diagrams}

This section considers some phase diagrams that have unusual features and yet are considered acceptable.

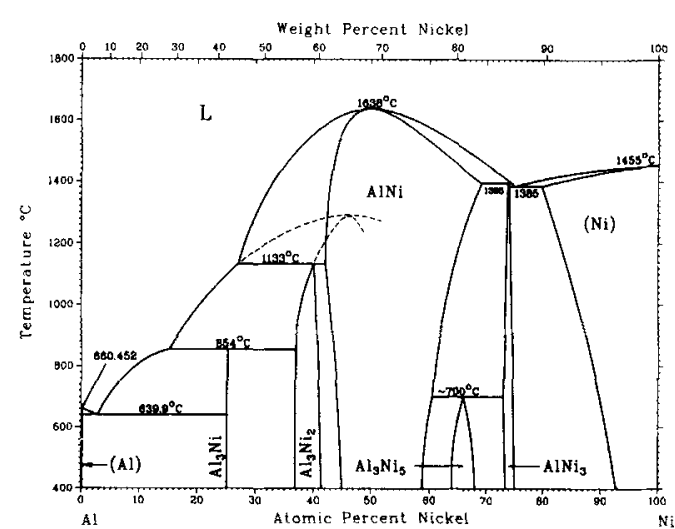

Fig. 28 Al-Ni phase diagram. From [90Sin]. Dashed lines added in this work.

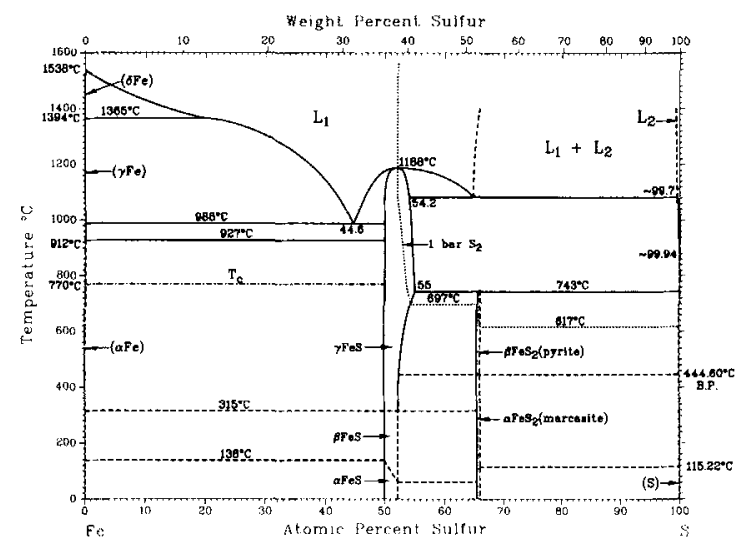

Fig. $29 \mathrm{Fe}-\mathrm{S}$ phase diagram. From [90Mas].

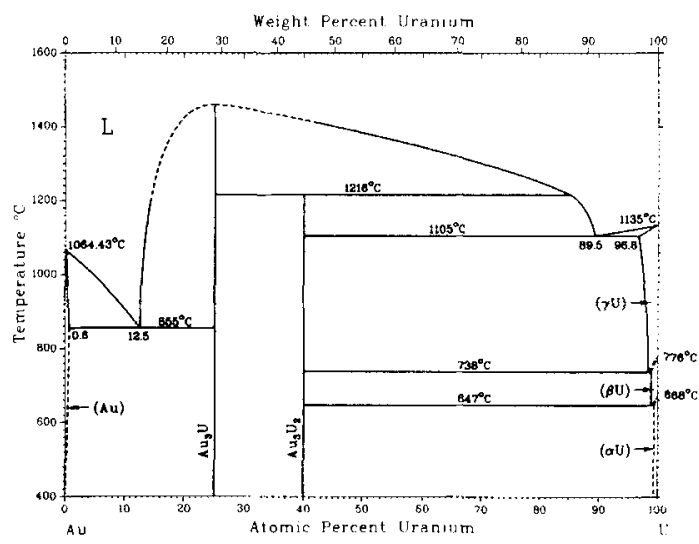

Fig. 30 Au-U phase diagram. From [860ka1].

\subsection{Apparent Absence of a Two-Phase Field}

In many binary phase diagrams of intra-rare earth systems [90Mas], quite often no two-phase fields are shown between the liquidus and the solidus (e.g., see Fig. 2). This is mainly because the difference in the melting points of two elements is small and both the liquidus and the solidus are nearly horizontal. In addition, the separation between the two lines is essentially too small to be recorded graphically. For example, between the liquidus and the solidus of the Er-Tm system, where the difference in the melting points of the two elements is only $16^{\circ} \mathrm{C}$, the maximum separation is -0.2 at.\% at any temperature, according to an ideal solution model. Clearly, a graphic phase diagram of an ordinary size cannot reveal such close separation. Thus, although the absence of a two-phase field appears to be an I-type error as in Fig. 1, it may be simply due to a two-phase field with an unresolvable width.

The larger the difference in the melting points, the more evident becomes the separation between the two lines. For example, the difference in the melting points of $\mathrm{Ho}$ and $\mathrm{Lu}$ is $189^{\circ} \mathrm{C}$, and the calculated maximum separation between the liquidus and the solidus is $\sim 3$ at.\%, which may be depicted graphically depending on the scale of the graphics used.

In the $\mathrm{Fe}-\mathrm{S}$ phase diagram (Fig. 29) [90Mas], the $(\delta \mathrm{Fe})$ to $(\gamma \mathrm{Fe})$ transformation temperature in alloys at $1365^{\circ} \mathrm{C}$ is lower than the $\delta \mathrm{Fe}$ to $\gamma \mathrm{Fe}$ allotropic transformation temperature of pure iron $\left(1394^{\circ} \mathrm{C}\right)$. In this case, the existence of a $(\delta \mathrm{Fe}) \leftrightarrow \mathrm{L}+(\gamma \mathrm{Fe})$ catatectic* reaction is required, and overlapping of three phase boundaries with infinite slopes between 1365 and $1394^{\circ} \mathrm{C}$ must be avoided. Assuming that the solubility of $\mathrm{S}$ in $(\gamma \mathrm{Fe})$ is negligible, the van't Hoff equation requires that the solubility of $S$ in $(\delta \mathrm{Fe})$ is about 0.1 at.\% (assuming $840 \mathrm{~J} / \mathrm{mol}$ [ $83 \mathrm{Cha}$ ] for the enthalpy of transformation of $\delta \mathrm{Fe}$ to $\gamma \mathrm{Fe}$ ). Because of this small solubility, the diagram with no apparent $(\delta \mathrm{Fe})+(\gamma \mathrm{Fe})$ two-phase field is acceptable.

\subsection{Asymmetric Liquidus}

If the shape of the liquidus is very asymmetric, with respect to the congruent melting point of a line compound, the situation merits

*[74Wag] proposed the term "catatectic" ("kata" = down, "tectic" = melt) for the solid $\rightarrow$ liquid + solid reaction on cooling. This reaction is also called "metatectic."

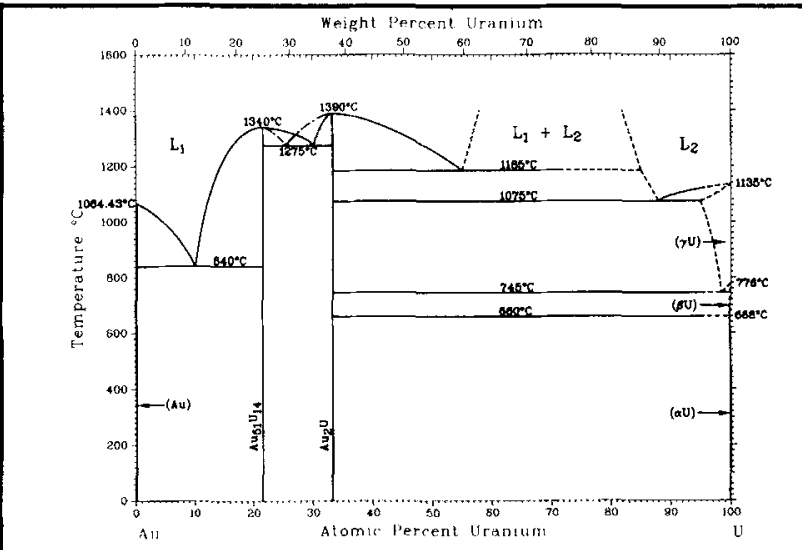

Fig. 31 Au-U phase diagram. Redrawn from [88Pal]. $-\cdot-\cdot-=$ present proposal. 
a closer examination. A liquidus with a strongly asymmetric form is unlikely, because an abrupt change in the composition dependence of the Gibbs energy of the liquid phase would be required at the composition of the compound without any obvious reasons. This situation may be illustrated with reference to the Au-U system. In the phase diagram shown in Fig. 30 [860ka1], the proposed liquidus of $\mathrm{Au}_{3} \mathrm{U}$ is strongly asymmetric and a rather

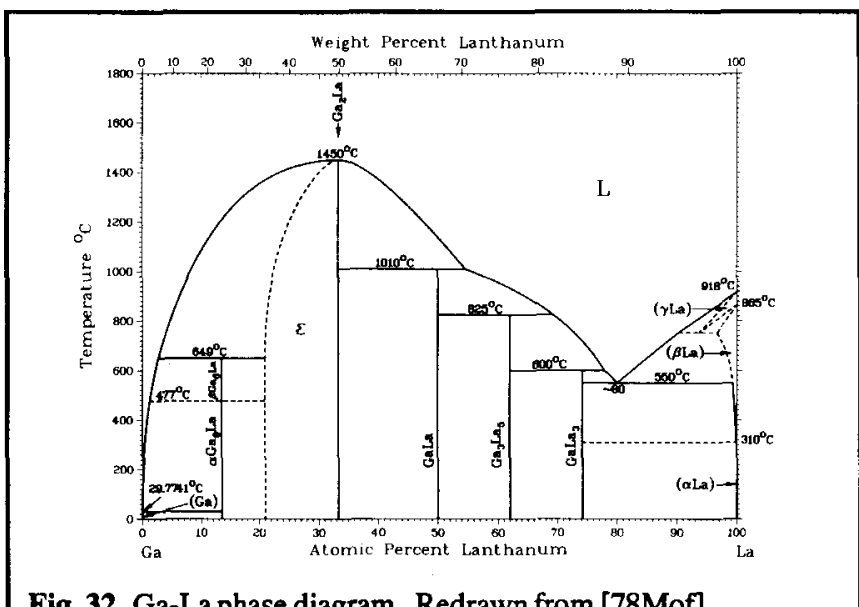

Fig. 32 Ga-La phase diagram. Redrawn from [78Mof].

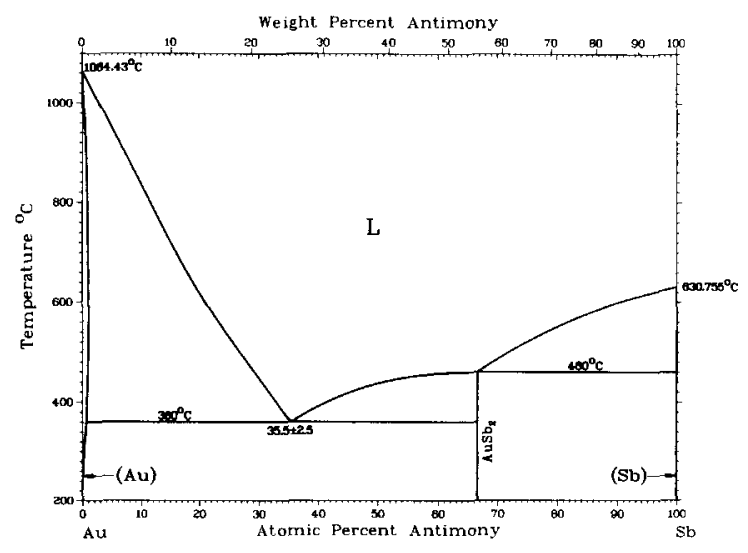

Fig. $33 \mathrm{Au}-\mathrm{Sb}$ phase diagram. From [84Oka].

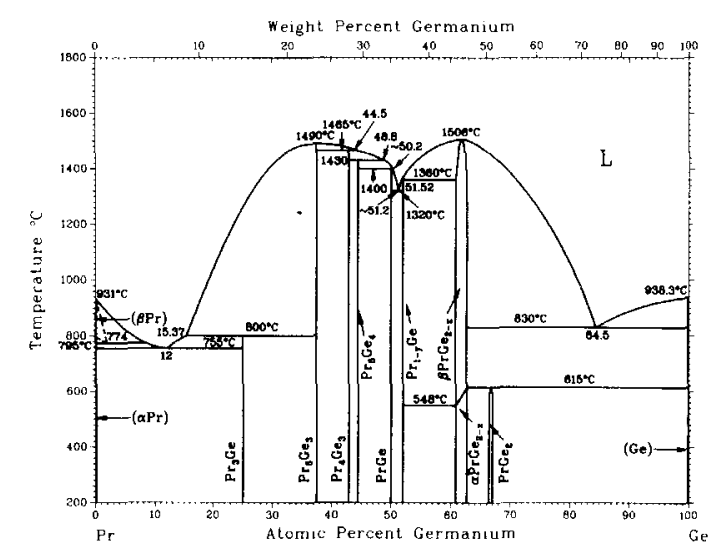

Fig. 34 Pr-Ge phase diagram. From [89Gok]. abrupt change of slope is required below $1216^{\circ} \mathrm{C}$ to avoid the $E$ type error in Fig. 1. Accordingly, the validity of this liquidus was questioned and other possible diagrams were proposed by [860ka2]. Figure 31 shows the Au-U diagram revised by [ $88 \mathrm{Pal}$ ], in which identifications of intermetallic compounds were corrected. However, the problem of asymmetry of the liquidus between $\mathrm{Au}_{51} \mathrm{U}_{14}$ and $\mathrm{Au}_{2} \mathrm{U}$ remains. The present authors speculate that the $\mathrm{L} \leftrightarrow \mathrm{Au}_{51} \mathrm{U}_{14}+\mathrm{Au}_{2} \mathrm{U}$ eutectic composition may actually be located closer to $\mathrm{Au}_{51} \mathrm{U}_{14}$, as shown with the dot-dashed lines.

As a general feature, if the liquidus is shown, by strong experimental evidence, to be asymmetric around a congruent melting point, most likely the compound also has asymmetric solid solubility. To illustrate, Fig. 32 shows valid asymmetric liquidus and solidus of $\mathrm{Ga}_{2} \mathrm{La}$ [78Mof].

\subsection{Phase Diagrams with an Apparent $R$-Error Appearance}

Five phase boundaries appear to be meeting at the melting point of $\mathrm{AuSb}_{2}$ in the $\mathrm{Au}$-Sb phase diagram (Fig. 33) [84Oka], suggesting an $R$-type error. (Note that the borders of the "line" compound $\mathrm{AuSb}_{2}$ should be counted as two solvus lines.) In this case, the melting point of $\mathrm{AuSb}_{2}$ and the invariant temperature (eutectic or peritectic) coincided within the experimental accuracy. Hence the apparent, but in this case not erroneous, type $R$ appearance is acceptable. This situation frequently occurs in binary phase diagrams.

\subsection{Apparent Four-Phase Equilibrium}

According to Fig. 1, a four-phase equilibrium is the $S$-type error. Therefore, the reaction at $615^{\circ} \mathrm{C}$ involving $\beta \operatorname{PrGe}_{2-x}, \alpha \operatorname{PrGe}_{2-x}$, $\mathrm{PrGe}_{2}$, and $(\mathrm{Ge})$ on the $\mathrm{Ge}$-rich side of the $\mathrm{Pr}-\mathrm{Ge}$ phase diagram (Fig. 34). [89Gok] apparently violates the phase rule. However, as mentioned in [89Gok], this is a coincidence of temperatures of the polymorphic transformation of $\mathrm{PrGe}_{2-x}$ and the peritectoid formation of $\mathrm{PrGe}_{2}$. The more serious problem in this diagram is the deep $\mathrm{L} \leftrightarrow \operatorname{PrGe}+\operatorname{Pr}_{1-y} \mathrm{Ge}$ eutectic reaction, because to accommodate this, liquidus boundaries with abrupt changes of curvature are required for $\mathrm{Pr}_{5} \mathrm{Ge}_{4}$ and $\beta \mathrm{PrGe}_{2-x}$ in the (upper) metastable equilibrium range. Mixed degrees of curvature of the liquidus boundaries in this range also pose a problem. Interestingly, an apparent violation of the phase rule in this system may be

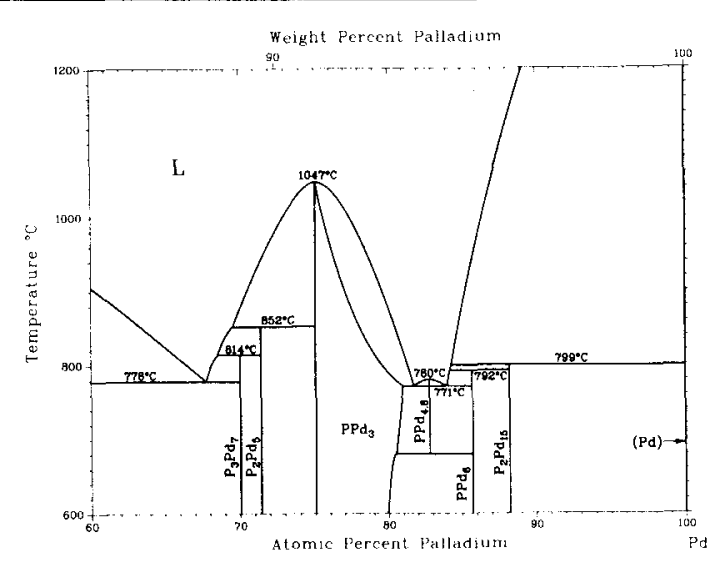

Fig. 35 Partial P.Pd phase diagram. From [910ka]. 
explained, but an apparent nonviolation is nevertheless more troublesome.

\subsection{Apparent Five-Phase Equilibrium}

In the P-Pd phase diagram (Fig. 35) [910ka], five phases, $\mathrm{PPd}_{3}$, $\mathrm{L}, \mathrm{PPd}_{4.8}, \mathrm{~L}$, and $\mathrm{PPd}_{6}$ appear to be in equilibrium. This is because two eutectic temperatures on either side of $\mathrm{PPd}_{4.8}$ have practically the same values, causing an appearance of the five-phase equilibrium. Hence, within the resolution scale of the graphics employed and the present experimental information, nothing is wrong with this diagram.

\subsection{Pointed Liquidus}

Some exceptions concerning the $Q$-type problem are mentioned here. For example, in the Cd-Te phase diagram (Fig. 36) [89Sha], the liquidus at the melting point of CdTe appears to be more pointed than ordinary congruent melting points of binary metalmetal systems. These types of pointed melting points are very common in halogen- and chalcogen-based systems, in which strong ionic bonds retain the non-dissociated molecular form of intermediate phase in the liquid state. The diagram appears to consist of Cd-CdTe and CdTe-Te subsystems, with CdTe behaving as an element. If the association of molecules in the liquid state is $100 \%$, the initial slope of the CdTe end can be derived

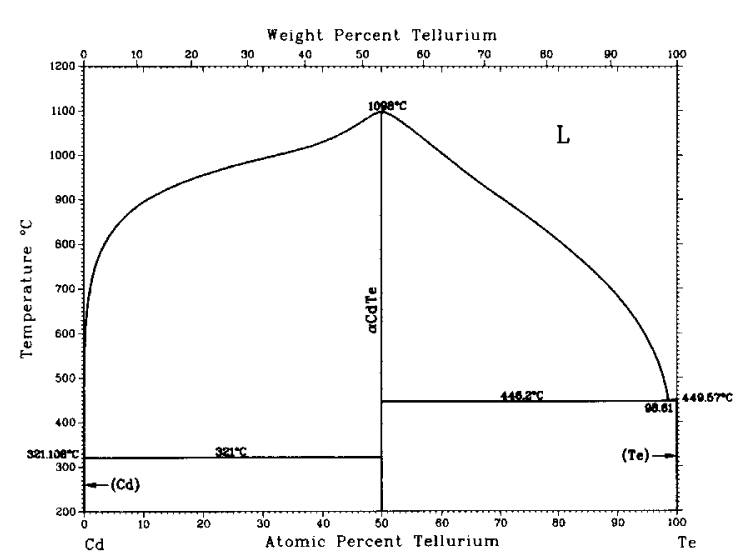

Fig. $36 \mathrm{Cd}$-Te phase diagram. From [89Sha].

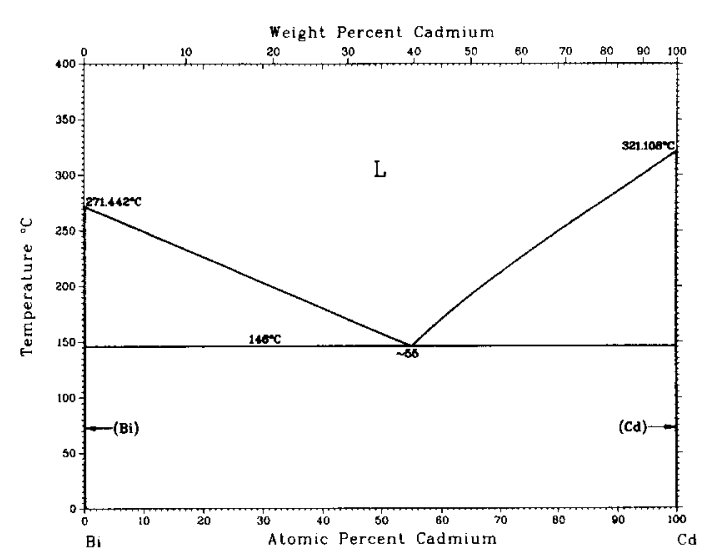

Fig. 37 Bi-Cd phase diagram. From [88Mos]. using $\mathrm{Eq} 1$ and the enthalpy of fusion of CdTe. However, because the association cannot be $100 \%$ above $0 \mathrm{~K}$, the slope of liquidus at the melting point must be horizontal [89Sha]. However, for viewing the overall trend of the liquidus, the compound should be regarded as an element, allowing a pointed liquidus at the melting point.

\subsection{Straight-Line Liquidus}

Sometimes a phase diagram may appear as if it were constructed by employing only straight lines, as in the $\mathrm{Bi}-\mathrm{Cd}$ phase diagram (Fig. 37) [88Mos]. At first glance it might appear that the graphics used are too simple. However, as long as the initial slope satisfies the van't Hoff relation, a liquidus can be a nearly straight line. For example, the calculated contour of the liquidus line in Fig. 19 corresponding to the interaction parameter of $-10000 \mathrm{~J} / \mathrm{mol}$ deviates by only about $10^{\circ} \mathrm{C}$ from a straight line over a wide composition range between 0 and $\sim 80$ at.\%. Therefore, there can be cases when straight line construction may be quite compatible with a possible thermodynamic model of the existing phase relationships.

\subsection{Solid-to-Solid Transformation Other than Polymorphic or Order-Disorder Transformation}

The Ta-Al diagram (Fig. 38) [90Kat] does not involve any violation of the thermodynamic requirements summarized in Fig. 1. However, the following unusual situations are found in this diagram. (1) The relationship between $\mathrm{Ta}_{2} \mathrm{Al}$ and $\mathrm{TaAl}$ is unusual. When the $\mathrm{Ta}_{2} \mathrm{Al} /\left(\mathrm{Ta}_{2} \mathrm{Al}+\mathrm{TaAl}\right)$ solvus is extrapolated to the $\mathrm{TaAl}$ side (this can be considered if the formation of the liquid is suppressed), it eventually crosses the TaAl line. (At this point, the solvus would also have to show a horizontal slope to avoid the $Q$ type problem.) Very rarely in binary phase diagrams does a solid compound phase transform to a neighboring compound phase on heating or cooling. This is underscored by the fact that the Gibbs energy compound usually has its lowest value at or very near to its ideal stoichiometry. Therefore, the apparent transformation from $\mathrm{Ta}_{2} \mathrm{Al}$ to $\mathrm{TaAl}$ in the metastable state at the composition of $\mathrm{TaAl}$ is most unusual. (2) The melting behavior of $\operatorname{Ta}_{2} \mathrm{Al}$ is unusual. When the liquidus and the solidus of $\mathrm{Ta}_{2} \mathrm{Al}$ are extrapolated above $2100^{\circ} \mathrm{C}$, they must come together in contact horizontally at the projected congruent melting point. Because abrupt changes of

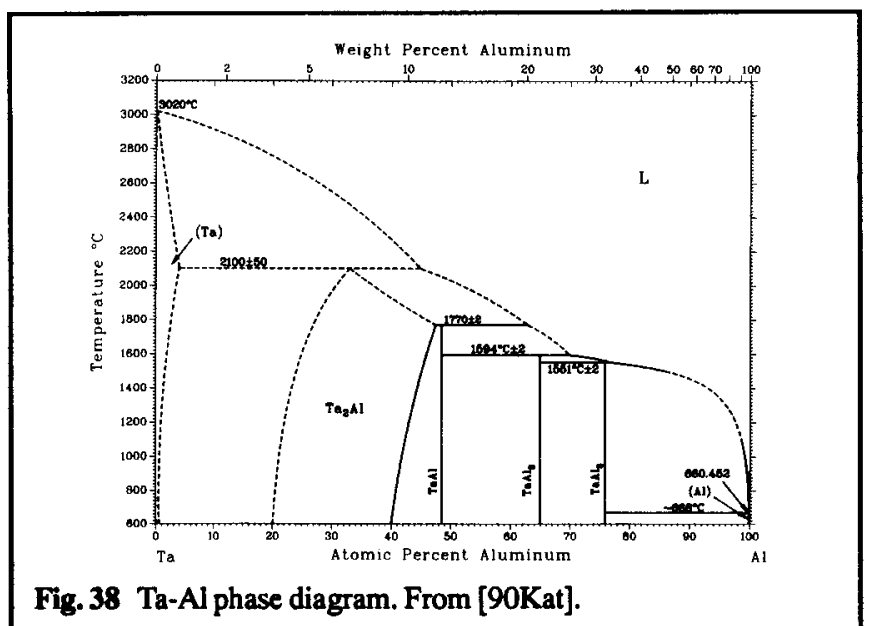


slopes must be avoided, the projected congruent melting point appears to occur at about 20 at.\% $\mathrm{Al}$, i.e., quite far away from the $\mathrm{Ta}_{2} \mathrm{Al}$ stoichiometry. Considering these two points, the projected form of the wide phase field of $\mathrm{Ta}_{2} \mathrm{Al}$ is probably incorrect. Possibly, $\mathrm{TaAl}$ does not exist (no report on its crystal structure is available) and the designation of $\mathrm{Ta}_{2} \mathrm{Al}$ does not seem appropriate.

\section{Suspicious or Erroneous Diagrams with Seemingly Acceptable Appearance}

\subsection{Retrograde Solubility of Gas in the Liquid}

When a liquid phase is in equilibrium with a gas phase, the liquid generally dissolves a diminishing amount of the gas phase with increasing temperatures, until the dissolved amount correctly becomes 0 at. \% at the boiling point. (This retrograde type of solubility is common in solid phases, and it is sometimes confused with a eutectic reaction.) Phase diagrams that require retrograde solubility of the gas phase with respect to the liquid phase are occasionally found in the literature, as shown for the B-Bi system (Fig. 39) [78Mof]. The expected solubility trend of the gas in the liquid is frequently confusing, and often the requirement that the solubility should diminish to zero at the boiling point is not realized.

\subsection{Apparent Single-Phase Field Between Two Elements with Different Crystal Structures}

At first glance, the solidus of the Lu-Th phase diagram (Fig. 40) [86Mas] renders no problem. However, the labeling of the $(\beta T h, \mathrm{Lu})$ phase is incorrect, because the bcc $\beta$ Th and cph Lu cannot form a continuous phase field between them. Hence, either a high-temperature boc form of Lu exists, or some vital portions of the phase diagram are missing.

The above example emphasizes that when a clear conflict with thermodynamical or crystal structure considerations arises, uncertain portions should be either drawn with dashed lines, or omitted altogether in order to emphasize the existing uncertainty.

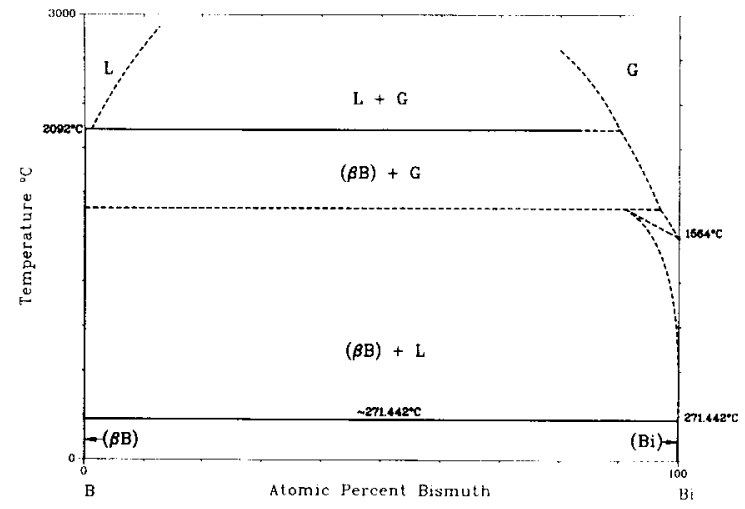

Fig. 39 B-Bi phase diagram. Redrawn from [78Mof].

\section{Summary and Conclusions}

Figure 41 represents a composite hypothetical diagram highlighting many of the unlikely phase relationships (points $a-p$ ) that have been described above. The problem at each point is discussed briefly in the Appendix.

This article provides a general set of guidelines for checking the consistency of phase diagrams encountered in binary systems. In order to understand the various subtleties of phase diagram construction, the reader should redraw Fig. 41 so that no errors remain (and only then consult the Appendix). The corrections need only be slight in order to achieve this goal. Nevertheless, if an anomaly is detected and corrected in a phase diagram, by checking the thermodynamic soundness and by using the criteria mentioned above, the correct diagram will not be achieved merely from the checking and reconciling of the boundaries. The ultimate diagram can be found only through experimental data obtained under proper equilibrium conditions and over sufficiently wide temperature and composition variations. For this reason, some of the diagrams in [90Mas] still involve portions that are thermodynamically improbable. The more likely trends discussed in this article have not been appllied to them for fear that they would depart further from the ultimate correct boundaries. The obvious next step in the development of a better understanding of phase diagram construction is a more systematic comparison between the evolution of the various features discussed above and the quantitative changes needed in the parameters involved in the thermodynamic functions that cause such features. In one or two examples, we have provided such quantitative comparisons in the present work. Increasing publications in the literature also show that both experimental and theoretical progress is rapidly taking place in this field. The publication of [90Mas] should further speed up this progress.

We apologize to the authors of unlikely phase diagrams quoted above for selecting their specific omissions or difficulties in the proposed phase diagrams. Some errors may have been committed inadvertently in the process of drawing, or data transcription, or digitization for graphical reproduction. Also, some diagrams have already been corrected or modified in subsequent publications, precisely to avoid the difficulties discussed in this article.

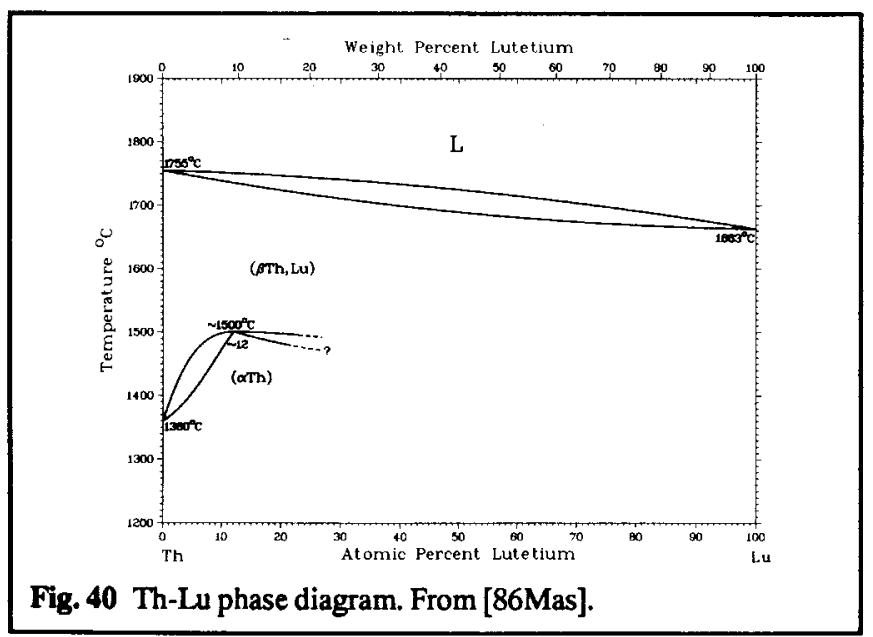




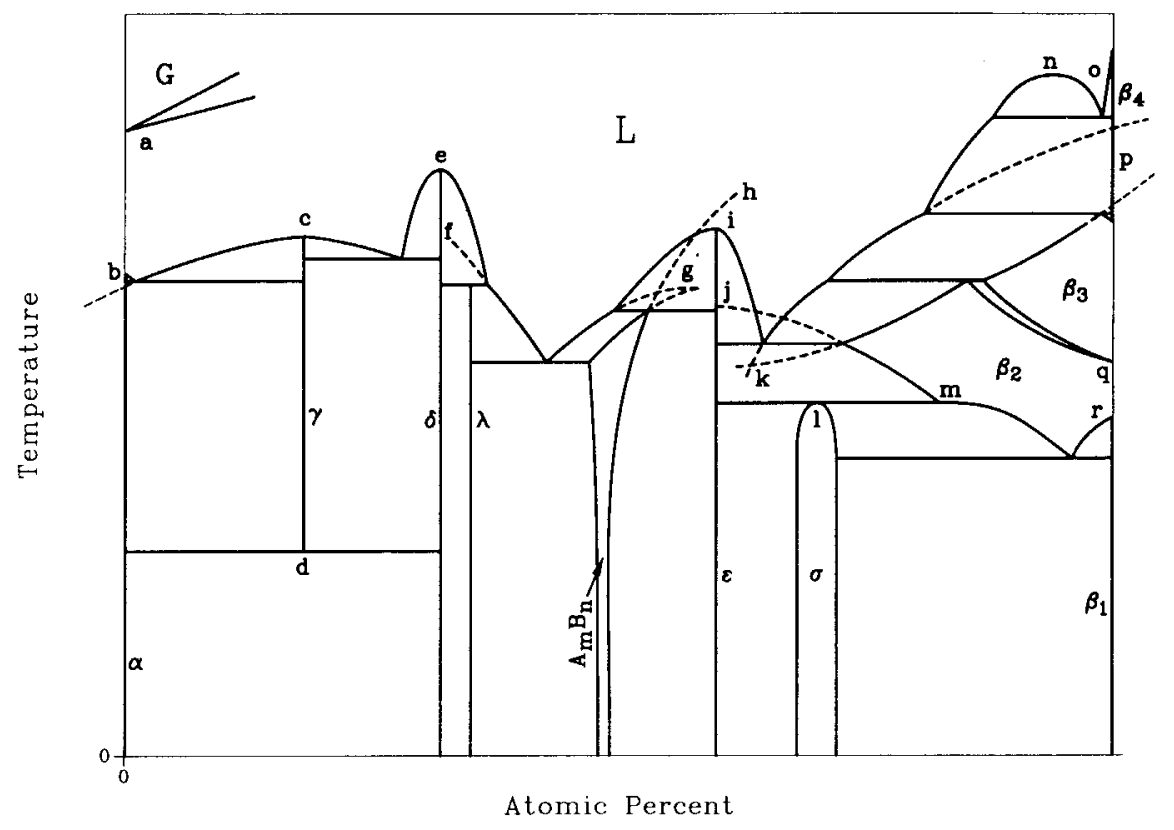

Fig. 41 Hypothetical phase diagram indicating improbable phase boundaries. Note that the phase rules are not violated explicitly.

\section{Acknowledgments}

We wish to acknowledge with thanks the partial support from the National Science Foundation (DMR 89-15875), which has made T.B. Massalski's long range interest and research related to phase diagrams possible.

\section{Cited References}

56Rhi: F.N. Rhines, Phase Diagrams in Metallurgy, McGraw-Hill, New York, 340p(1956).

57Bec: L.H. Beck and C.S. Smith, "Copper-Zinc Constitution Diagram, Redetermined in the Vicinity of the Beta Phase by Means of Quantitative Metallography," Trans. AIME, 194(10), 1079-1083(1957).

58Han: M. Hansen and $\mathrm{K}$. Anderko, Constitution of Binary Alloys, originally published by McGraw-Hill, reprinted and available from Genium Publishing Corporation, 1145 Catalyn Street, Schenectady, New York 12303(1958).

65Ell: R.P. Elliot, Constitution of Binary Alloys, First Supplement, originally published by McGraw-Hill, reprinted and available from Genium Publishing Corporation, 1145 Catalyn Street, Schenectady, New York 12303(1965).

66Pri: A. Prince, Alloy Phase Equilibria, Elsevier Publ. Co., London (1966).

68Gor: P. Gordon, Principles of Phase Diagrams in Material Systems, McGraw-Hill, New York, 232p(1968).

69Rud: E. Rudy, Ternary Phase Equilibria in Transition Metal-BoronCarbon-Silicon Systems, Part V. Compendium of Phase Diagram Data, Air Force Materials Laboratory, Air Force Systems Command, Wright-Patterson Air Force Base, OH, 214-215 (1969).

69Shu: F.A. Shunk, Constitution of Binary Alloys, Second Supplement, originally published by McGraw-Hill, reprinted and available from Genium Publishing Corporation, 1145 Catalyn Street, Schenectady, New York 12303 (1969).
70Thu: R. Thummel and W. Klemm, "Behavior of Alkali Metals in Metals of Group III B," Z.Anorg. Allg. Chem., 376, 44-63(1970).

73Hul: R. Hultgren, P.D. Desai, D.T. Hawkins, M. Gleiser, K.K. Kelley, and D.D. Wagman, Selected Values of the ThermodynamicProperties of the Elements, American Society for Metals, Metals Park, OH (1973).

74Wag: S. Wagner and D.A. Rigney, "Binary Systems Involving the 'Catatectic' Reaction Solid 1 Cooling $\leftrightarrow$ Heating Solid 2 + Liquid," Metall. Trans., 5(10), 2155-2160(1974).

76Alc: C.B. Alcock, K.T. Jacobs, S. Zador, O. von Goldbeck, H. Nowotny, K. Seifert, and O. Kubaschewski, Zirconium, Physico-Chemical Properties of Its Compounds and Alloys, O. Kubaschewski, Ed., Atomic Energy Review Special Issue No. 6, International Atomic Energy Agency, Vienna(1976).

78Mof: W.G. Moffatt, Handbook of Binary Phase Diagrams, Genium Publishing Corporation, Schenectady, New York (1978) and Supplements.

81Goo: D.A. Goodman, J.W. Cahn, and L.H. Bennett, "The Centennial of the Gibbs-Konovalov Rule for Congruent Points," Bull. Alloy Phase Diagrams, 2(1), 29-34(1981).

81Nis: T. Nishizawa and M. Hasebe, "Computer Calculation of Phase Diagrams of Iron Alloys (1)," Tetsu to Hagane, 67(11), 1887-1898 (1981).

81Smi: J.F. Smith, "The Sn-V (Tin-Vanadium) System," Bull. Alloy Phase Diagrams, 2(2), 210-214 (1981).

82Gsc: K.A. Gschneidner, Jr. and F.W. Calderwood, "The Ce-Pr (Cerium-Praseodymium) System," Bull. Alloy Phase Diagrams, 3(2), 187-188(1982).

83Cha: M.W. Chase, "Heats of Transition of the Elements," Bull. Alloy Phase Diagrams, 4(1), 123-124(1983).

84Nay: A.A. Nayeb-Hashemi and J.B. Clark, "The Mg-Sb (Magnesium-Antimony) System," Bull Alloy Phase Diagrams, 5(6), 579 584(1984). 
840ka: H. Okamoto and T.B. Massalski, "The Au-Sb(Gold-Antimony) System," Bull. Alloy Phase Diagrams, 5(2), 166-171 (1984).

84Ole: R.W. Olesinski and G.J. Abbaschian, "The C-Ge (Carbon- Germanium) System," Bull. Alloy Phase Diagrams, 5(5), 484-486 (1984).

84Pal: A. Palenzona, P. Manfrinetti, and S. Cirafici, "The Th-In Phase Diagram,"J. Less-CommonMet, 97, 231-236(1984).

86Mas: T.B. Massalski, J.L. Murray, L.H. Bennett, and H. Baker, Binary Alloy Phase Diagrams, 1st ed., American Society for Metals, Metals Park, OH (1986).

860ka1: H. Okamoto and T.B. Massalski, "The Au-U(Gold-Uranium) System," Bull. Alloy Phase Diagrams, 7(6), 532-535(1986).

860ka2: H. Okamoto and T.B. Massalski, "Phase Relationships and Thermodynamic Modeling in Several Binary Systems Based on Gold," Noble Metal Alloys: Phase Diagrams, Alloy Phase Stability, ThermodynamicAspects, Properties and Special Features, T.B. Massalski, W.B. Pearson, L.H. Bennett, and Y.A. Chang, Ed., Metallurgical Society, Inc., Warrendale, PA, 265-288(1986).

87Alc: C.B. Alcock, P.G. Komorowski, V.P. Itkin, and M. Itkin, Micro Tbank, University of Toronto, Toronto, Canada (1987).

88Itk: V.P. Itkin, C.B. Alcock, P.J. van Ekeren, and H.A. J. Oonk, "The Al-Ca (Aluminum-Calcium) System," Bull. Alloy Phase Diagrams, $9(6), 652-657(1988)$.

88Mos: Z. Moser, J. Dutkiewicz, L. Zabdyr, and J. Salawa, "The Bi-Cd (Bismuth-Cadmium) System," Bull. Alloy Phase Diagrams, 9(4), 445-448(1988).

88Pal: A. Palenzona and S. Cirafici, "The Phase Diagram of the U-Au System,"J. Less-CommonMet., 143, 167-171 (1988).
88Sub: P.R. Subramanian and D.E. Laughlin, "The Cu-Lu (CopperLutetium) System.," Bull. Alloy Phase Diagrams, 9(3a), 358-361 (1988).

89Gok: A.B. Gokhale, A. Munitz, and G.J. Abbaschian, "The Ge-Pr (Germanium-Praseodymium) System," Bull. Alloy Phase Diagrams, 10(3), 241-246(1989).

89How: J.M. Howe, "The Al-Se (Aluminum-Selenium) System," Bull. Alloy Phase Diagrams, 10(6), 650-652(1989).

89Sha: R.C. Sharma and Y.A. Chang, "Thermodynamic Analysis and Phase Equilibria Calculations for the Cd-Te, Cd-Se, and Cd-S Systems,"J.Electrochem. Soc., 136(5), 1536-1542(1989).

90Car: O.N. Carlson, "The Al-B (Aluminum-Boron) System," Bull. Alloy Phase Diagrams, 11(6), 560-566(1990).

90Din: A.T. Dinsdale, "SGTE Pure Elements Transformation Data," T13-T15, reprinted in [90Mas].

90Kat: U.R. Kattner, "Al-Ta (Aluminum-Tantalum)," reprinted in [90Mas].

90Mas: T.B. Massalski, H. Okamoto, P.R. Subramanian, and L. Kacprzak, Binary Alloy Phase Diagrams, 2nd ed., ASM International, Materials Park, OH (1990).

90Sin: M.F. Singleton, J.L. Murray, and P. Nash, "Al-Ni (AluminumNickel)," in [90Mas], 181, 183-184 (1990).

90Ven1: M. Venkatraman and J.P. Neumann, "The Cr-Os (ChromiumOsmium) Phase Diagram," Bull. Alloy Phase Diagrams, 11(1), 8-11 (1990).

90Ven2: M. Venkatraman and J.P. Neumann, "The C-Cr (CarbonChromium) Phase Diagram," Bull. Alloy Phase Diagrams, 11(2), 152-159(1990).

910ka: H. Okamoto, "The P-Pd(Phosphorus-Palladium) System, to be published in J. Phase Equilibria (1991).

\section{Appendix}

Figure a1 illustrates an error-free Fig. 1 (see "1.1 Typical Phase Rule Violations").

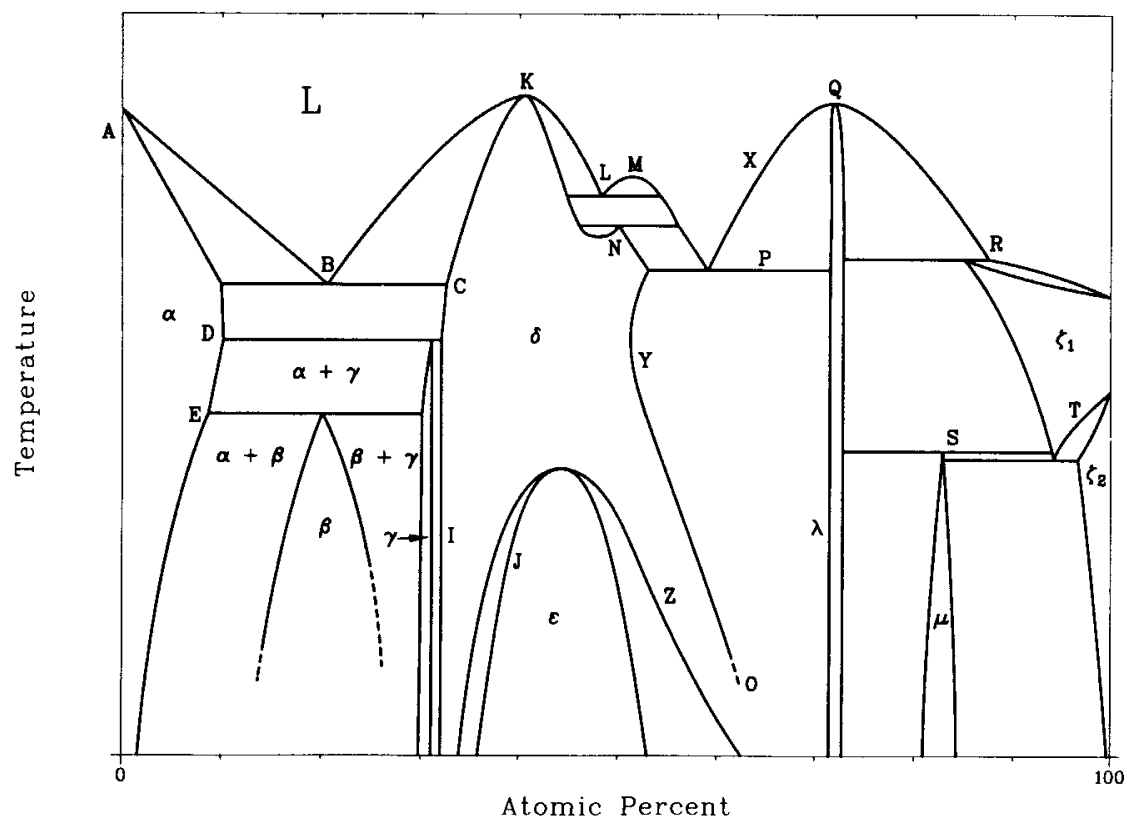

Fig. a1 Possible error-free phase diagram of Fig. 1. 


\section{Section I: Basic and Applied Research}

The problems at each point in Fig. 41 are as follows:

$a: \mathrm{G}+\mathrm{L}$ two-phase field is too narrow.

$b$ : Extrapolation of liquidus should not cross the 0 at.\% line.

c: The liquidus of $y$ is too flat in comparison with the liquidus of $\delta$ at point $e$.

$d$ : A compound with a flat liquidus is stable and will not decompose at low temperatures.

$e:$ Liquidus is too sharp in comparison with the point $c$.

$f$ : Extrapolation of liquidus of $\lambda$ must have a peak at the composi tion of $\lambda$.

$g: A_{m} B_{n}$ compound with the congruent melting point far away from its stoichiometric composition.

$h$ : A phase field of a compound extending over a neighboring phase.

$i$ : Asymmetric liquidus. $j$ : The transformation temperature of $\varepsilon$ to $\beta_{2}$ should be higher than the melting point of $\varepsilon$. Otherwise, the $\beta_{2}$ phase is stable above point $j$.

$k$ : Extrapolation of two boundaries of $L+\beta_{2}$ two-phase field should not cross.

l: The boundary of $\sigma$ phase should not have a round maximum at the peritectoid temperature.

$m$ : The slope is too flat to have a maximum point at the composition of $\sigma$.

$n$ : The miscibility gap is too close to the edge of a phase diagram.

$o$ : The liquidus slope is too steep.

$p$ : Extrapolation of two boundaries of $\mathrm{L}+\beta_{3}$ should cross at the 100 at.\% line, not at $>100$ at.\%.

$q$ : Two phase boundaries should have different initial slopes.

$r$ : The slopes of two phase boundaries are too far apart. 